\title{
Afterload promotes maturation of human induced pluripotent stem cell derived cardiomyocytes in engineered heart tissues
}

\author{
Andrea Leonard ${ }^{\mathrm{a}, \mathrm{d}, \mathrm{e}}$, Alessandro Bertero ${ }^{\mathrm{b}, \mathrm{d}, \mathrm{e}}$, Joseph D. Powers ${ }^{\mathrm{c}, \mathrm{d}, \mathrm{e}}$, Kevin M. Beussman ${ }^{\mathrm{a}, \mathrm{d}, \mathrm{e}}$, \\ Shiv Bhandari ${ }^{\mathrm{f}}$, Michael Regnier ${ }^{\mathrm{c}, \mathrm{d}, \mathrm{e}}$, Charles E. Murry ${ }^{\mathrm{b}, \mathrm{c}, \mathrm{d}, \mathrm{e}, \mathrm{f}, \mathrm{g}, * *}$, Nathan J. Sniadecki ${ }^{\mathrm{a}, \mathrm{c}, \mathrm{d}, \mathrm{e}, *}$ \\ a Department of Mechanical Engineering, University of Washington, Seattle 98107, WA, USA \\ ${ }^{\mathrm{b}}$ Department of Pathology, University of Washington, Seattle 98109, WA, USA \\ ${ }^{\mathrm{c}}$ Department of Bioengineering, University of Washington, Seattle 98107, WA, USA \\ d Center for Cardiovascular Biology, University of Washington, Seattle 98109, WA, USA \\ ${ }^{\mathrm{e}}$ Institute for Stem Cell and Regenerative Medicine, University of Washington, Seattle 98109, WA, USA \\ ${ }^{\mathrm{f}}$ Department of Medicine, University of Washington, Seattle 98195, WA, USA \\ ${ }^{\mathrm{g}}$ Division of Cardiology, University of Washington, Seattle 98195, WA, USA
}

\section{A R T I C L E IN F O}

\section{Keywords:}

Human induced pluripotent stem cell-derived cardiomyocytes

Cardiac maturation

Engineered heart tissue

Afterload

Hypertrophy

\begin{abstract}
A B S T R A C T
Human induced pluripotent stem cell-derived cardiomyocytes (hiPSC-CM) grown in engineered heart tissue (EHT) can be used for drug screening, disease modeling, and heart repair. However, the immaturity of hiPSCCMs currently limits their use. Because mechanical loading increases during development and facilitates cardiac maturation, we hypothesized that afterload would promote maturation of EHTs. To test this we developed a system in which EHTs are suspended between a rigid post and a flexible one, whose resistance to contraction can be modulated by applying braces of varying length. These braces allow us to adjust afterload conditions over two orders of magnitude by increasing the flexible post resistance from 0.09 up to $9.2 \mu \mathrm{N} / \mu \mathrm{m}$. After three weeks in culture, optical tracking of post deflections revealed that auxotonic twitch forces increased in correlation with the degree of afterload, whereas twitch velocities decreased with afterload. Consequently, the power and work of the EHTs were maximal under intermediate afterloads. When studied isometrically, the inotropy of EHTs increased with afterload up to an intermediate resistance $(0.45 \mu \mathrm{N} / \mu \mathrm{m})$ and then plateaued. Applied afterload increased sarcomere length, cardiomyocyte area and elongation, which are hallmarks of maturation. Furthermore, progressively increasing the level of afterload led to improved calcium handling, increased expression of several key markers of cardiac maturation, including a shift from fetal to adult ventricular myosin heavy chain isoforms. However, at the highest afterload condition, markers of pathological hypertrophy and fibrosis were also upregulated, although the bulk tissue stiffness remained the same for all levels of applied afterload tested. Together, our results indicate that application of moderate afterloads can substantially improve the maturation of hiPSC-CMs in EHTs, while high afterload conditions may mimic certain aspects of human cardiac pathology resulting from elevated mechanical overload.
\end{abstract}

\section{Introduction}

Human induced pluripotent stem cell-derived cardiomyocytes (hiPSC-CMs) have numerous potential applications including drug screening, developmental and disease modeling, and cardiac regeneration [1]. However, such uses are currently limited by the immature nature of hiPSC-CM obtained from available in vitro differentiation protocols [2]. During development, cardiomyocyte maturation occurs simultaneously with progressive increases in mechanical loading. This results from both changing myocardial tissue elasticity [3] due to extracellular matrix production, and to increased contractile resistance due to rising blood pressure [4]. However, standard plastic tissue culture dishes for in vitro studies are orders of magnitude stiffer than native myocardium $\left(\sim 10^{6} \mathrm{kPa}\right.$ versus $5-15 \mathrm{kPa}$ for normal heart tissue [5]), and cannot mimic the dynamic changes in loading present in vivo during development. Thus, the use of engineered

\footnotetext{
Abbreviations: hiPSC-CM, human induced pluripotent stem cell-derived cardiomyocyte; EHT, engineered heart tissue; PDMS, polydimethylsiloxane; PLA, polylactic acid; RT-qPCR, real time quantitative reverse transcription polymerase chain reaction

* Correspondence to: N. J. Sniadecki, University of Washington, 850 Republican Street, Brotman Building Room 442, Seattle, WA 98109, USA.

** Correspondence to: C. E. Murry, University of Washington, 850 Republican Street, Brotman Building Room 453, Seattle, WA 98109, USA.

E-mail addresses: murry@uw.edu (C.E. Murry),nsniadec@uw.edu (N.J. Sniadecki).
} 
heart tissue (EHT) platforms [6,7] that can vary afterload may allow for tunable loading at both the cellular and whole tissue level, and help to fulfill the potential of hiPSC-CMs.

In vivo, mechanical load during the cycle of a heartbeat is typically split in two phases: (1) preload, which refers to the wall stress or sarcomere length at the end of diastole, and (2) afterload, referring to the systolic load on the left ventricle during a contraction, which stems primarily from the blood pressure [8]. To better mimic native myocardium, most EHT platforms apply some level of uniaxial tension to generate tissues with an aligned myofibril structure [9-11]. Further application of cyclic stretch, which many consider comparable to varying preload, has been shown to improve cardiomyocyte maturation in EHTs [12-20]. However, the role of afterload on cardiomyocyte function and maturation within EHTs has been less explored [21-23]. Earlier work used neonatal rat cardiomyocytes in EHTs [21,22], as opposed to developing hiPSC-CMs which more closely resemble an embryonic developmental stage [2]. Taken together with the important physiological differences between these two species, it is unclear if earlier findings can be extrapolated to the clinically relevant human model. Furthermore, previous focus has been on testing two discrete afterloads as opposed to a more comprehensive range of loading conditions. While mechanical stimuli are known to be important mediators of physiological cardiac hypertrophy [24], sustained changes to the in vivo mechanical environment can also result in pathological hypertrophy and subsequent heart failure [8,25,26]. Overall, an existing challenge is to better understand the various possible benefits and adverse effects of mechanical stimuli in vitro, which is valuable to model both maturation and cardiac pathology.

Here, we assessed the role of afterload on the performance and maturation of EHTs containing hiPSC-CMs by varying the resistance to contraction (Fig. 1 and Movie S1). We designed a platform that allowed us to test an extensive range of afterload conditions that permitted auxotonic contractions, with the highest afterload resulting in a near isometric state. We include data from EHTs in unloaded (UL) conditions for comparison with our afterload conditions and with existing literature. After three weeks in culture, we assessed the auxotonic contractile performance of EHTs in their respective culture conditions, and subsequently compared their force generating capacity under isometric conditions. We also assessed structural maturation, calcium handling properties, and changes in gene expression resulting from increased afterload. Our results show that applied afterload can improve structure and functional maturation of hiPSC-CM, but that the benefits in contractile function plateau at a moderate afterload $(0.45 \mu \mathrm{N} / \mu \mathrm{m})$ and potentially adverse effects begin to appear at very high afterload $(9.2 \mu \mathrm{N} / \mu \mathrm{m})$. This work further emphasizes the need for suitable afterloads conditions in generating physiologically mature EHTs.

\section{Materials and methods}

\subsection{EHT platform design and fabrication}

The design of our post system and its adaptation to tissue culture dishes are shown in Fig. 1 and Fig. S1. Racks of polydimethylsiloxane (PDMS) posts were fabricated by pouring uncured PDMS (Sylgard 184 mixed at a 1:10 curing agent to base ratio) into a 4-part acrylic mold. Racks consisted of six pairs of posts that could be fit in a row of 24-well plate. Glass capillary tubes (Drummond, Cat \#1-000-0500) were cut to length and inserted into one side of the mold before curing to render one post rigid in each pair. Posts were baked overnight at $65{ }^{\circ} \mathrm{C}$ before being removed from the molds. Fabricated posts were $22 \mathrm{~mm}$ long and $1.5 \mathrm{~mm}$ in diameter with a cap structure $(0.5 \mathrm{~mm}$ thick and $2.0 \mathrm{~mm}$ diameter) to aide in the attachment of EHTs. The center-to-center post spacing was $8 \mathrm{~mm}$. Braces of different lengths were 3D printed in polylactic acid, PLA (MakerGear, natural $1.75 \mathrm{~mm}$ diameter filament), and three $1.5 \mathrm{~mm}^{3}$ cube N42 neodymium magnets (Magnakoys, MM$116 \times 116 \times 116)$ were glued into the base of each brace half. The magnets held the brace halves in place around a pair of posts to shorten the effective length of the posts and thereby increase their bending stiffness. The bending stiffness of the flexible posts with and without the braces was calibrated using a micromanipulator (InjectMan NI 2) with a glass pipette of known stiffness $(0.28 \mu \mathrm{N} / \mu \mathrm{m})$ following a previously described procedure [27]. Braces were designed to prop up the post rack from the bottom of the dish when placed upside down into the 24well plate rims, and a boundary wall was 3D printed to maintain sterility (Fig. S1 A-D). Prior to casting EHTs, all 3D printed parts and PDMS posts were sterilized in a UVO Cleaner (Jetlight, No. 342) for 7 min, submerged in $70 \%$ ethanol, and rinsed with sterile deionized water.
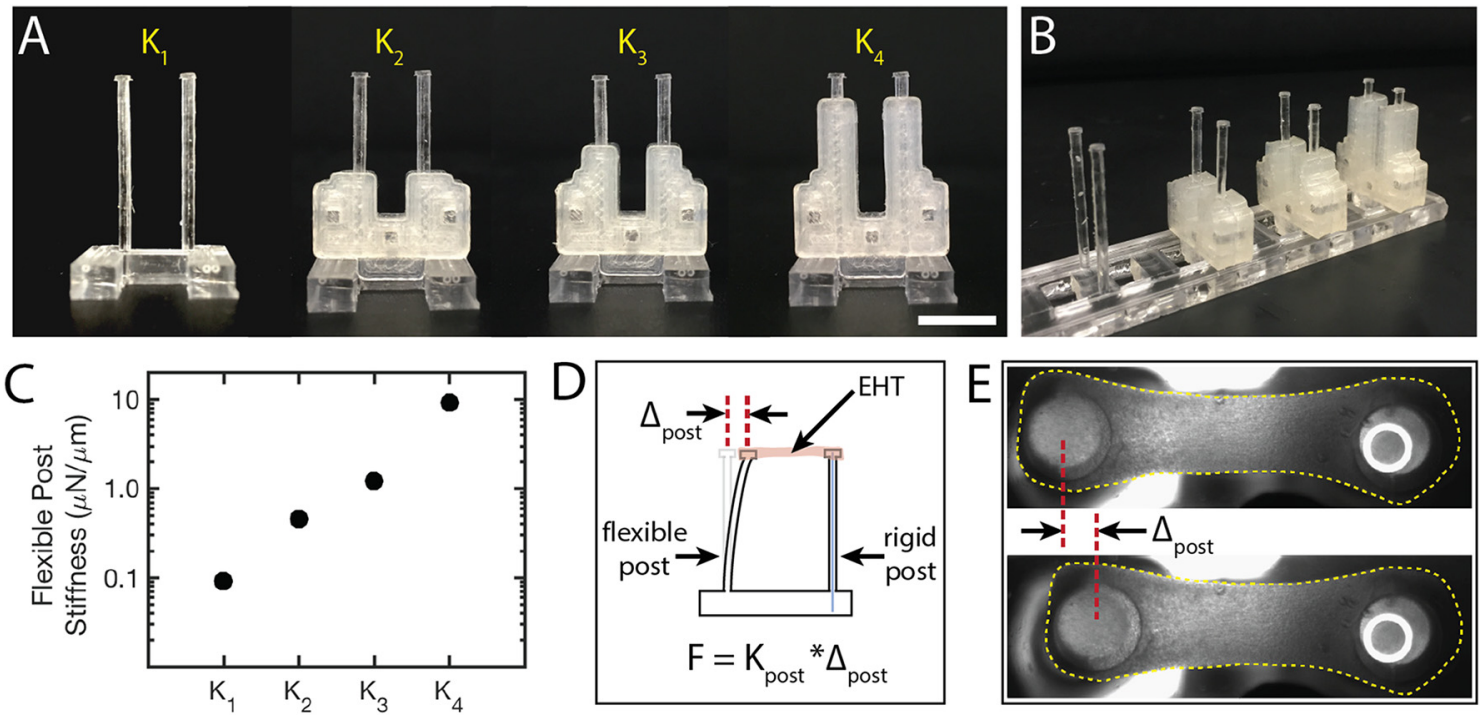

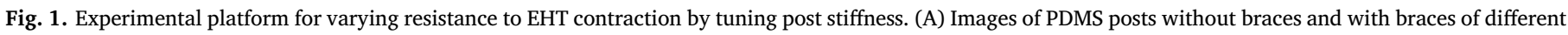

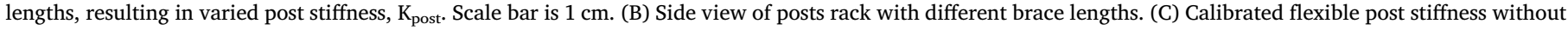

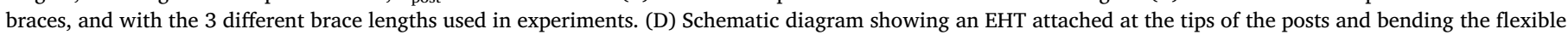

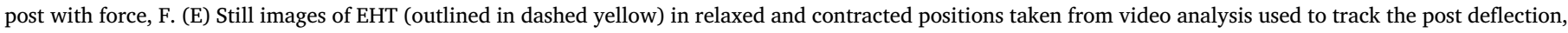
$\Delta_{\text {post }}$ (For interpretation of the references to colour in this figure legend, the reader is referred to the web version of this article.) 


\subsection{Cell culture and EHT generation}

Undifferentiated hiPSCs from the IMR90 line (WiCell) were maintained in mTeSR1 (Stemcell Technologies) on tissue culture dishes coated with a $60 \times$ dilution of Matrigel (Corning) in DMEM/F12 (Gibco). Standard G-banding analysis was performed to confirm that the cells were karyotypically normal. Cardiomyocytes were derived following the ABCX (activin A, BMP4, and small molecule Wnt modulators Chiron 99021 and Xav939) monolayer directed differentiation protocol as previously described [28]. Briefly, $1.3 \times 10^{6}$ undifferentiated cells were seeded in each well of a 6-well dish (coated with Matrigel) in mTeSR containing $10 \mu \mathrm{M}$ Y-27632 ROCK Inhibitor and $1 \mu \mathrm{M}$ Chiron 99021 (Tocris). The following day (referred to as day 0 on the differentiation timeline), the media was changed to RPMI media (Gibco) plus B-27 without insulin (Life Technologies), Matrigel, and $100 \mathrm{ng} / \mathrm{mL}$ Activin A (R\&D Systems). Eighteen hours later (day 1), the media was changed to RPMI containing B-27 without insulin, $5 \mathrm{ng} / \mathrm{mL}$ BMP4 (R\&D Systems), and $1 \mu \mathrm{M}$ Chiron 99021. On day 3, media was changed to RPMI containing B-27 without insulin and $1 \mu \mathrm{M}$ XAV939 (Tocris). On day 5, media was changed to RPMI containing B-27 without insulin. On day 7, the media was changed to RPMI containing B-27 with insulin and penicillin/streptomycin (Invitrogen), and was subsequently changed every 2-3 days.

Trypsin-dissociated hiPSC-CMs were frozen for stocks in CryoStor ${ }^{\circledast}$ cell cryopreservation media (Sigma-Aldrich) between 16 and 19 days after the start of differentiation. For experiments, CMs were thawed onto Matrigel coated dishes (at approximately $10 \times 10^{6}$ cells per $10-\mathrm{cm}$ dish) in RPMI plus B-27 with insulin and penicillin/streptomycin, which was supplemented with $10 \%$ FBS for the first $24 \mathrm{~h}$. The hiPSCCMs were allowed to recover for 5-7 days before EHT generation. All differentiations yielded high purity CMs ( $>90 \%$ ), as measured by flow cytometry after staining for cardiac troponin T (Fig. S2 C). Tissue constructs were generated following previously described protocols [29,30]. In short, rectangular $2 \% \mathrm{w} / \mathrm{vol}$ agarose/DPBS-casting molds (12 $\mathrm{mm}$ in length, $4 \mathrm{~mm}$ in width, and $\sim 4 \mathrm{~mm}$ in depth) were prepared in standard 24-well dishes by using custom 3D printed spacers as negative molds. PDMS posts were positioned upside down upon a 3D printed spacer in the middle of each well such that there was a $0.5 \mathrm{~mm}$ gap between the tip of the post and the bottom of the casting mold. Each tissue consisted of a $97 \mu \mathrm{L}$ fibrinogen-media solution $(89 \mu \mathrm{L}$ of RPMI containing B-27 with insulin; $5.5 \mu \mathrm{L}$ of $2 \times$ DMEM plus $20 \%$ FBS; and $2.5 \mu \mathrm{L}$ of $0.2 \mathrm{mg} / \mathrm{mL}$ bovine fibrinogen, Sigma-Aldrich) containing $5 \times 10^{5}$ hiPSC-CMs and $5 \times 10^{4}$ supporting HS27a human bone marrow stromal cells (ATCC), which was mixed with $3 \mu \mathrm{L}$ of thrombin (at $100 \mathrm{U} / \mathrm{mL}$, Sigma-Aldrich) just before pipetting into the agarosecasting mold. The EHT mixtures were incubated for $90 \mathrm{~min}$ at $37^{\circ} \mathrm{C}$, at which point the fibrin gels were sufficiently polymerized around the posts to be lubricated in media and transferred into a 24-well plate with fresh EHT media (RPMI containing B-27 plus insulin, penicillin/streptomycin, and $5 \mathrm{mg} / \mathrm{mL}$ aminocaproic acid, Sigma-Aldrich). EHTs were supplied with $2.5 \mathrm{~mL} /$ well of fresh EHT media three times per week.

\subsection{In situ force measurements}

In situ force measurements were taken for EHT contractions in a Tyrode's buffer with $1.8 \mathrm{mM} \mathrm{Ca}^{2+}$ at $37^{\circ} \mathrm{C}$. Videos of at least five contractions were recorded inside a heated chamber using a monochrome CMOS camera (Mightex, SMN-B050-U) with a board lens (The Imaging Source, TBL 8.4-2 5MP). The camera-lens configuration allowed for a capture rate of 65 frames per second (FPS) with $8.3 \mu \mathrm{m} /$ pixel resolution and a field of view of $1536 \times 400$ pixels, which was sufficient to capture images of the whole EHT from rigid to flexible post. A custom Matlab program was used to threshold the images and track the centroid of the flexible post relative to the centroid of the rigid post. Twitch force, velocity, power, and work were then calculated from the deflection of the flexible post and its bending stiffness, as previously described [27]. A custom-built 24-well plate with carbon electrodes (Fig. S1 E-F) was connected through an electrical stimulator (Astro Med Grass Stimulator, Model S88X) to provide biphasic field stimulation $(5 \mathrm{~V} / \mathrm{cm}$ for $10 \mathrm{~ms}$ durations) during imaging. For the experiments shown, EHTs were paced at $1.75 \mathrm{~Hz}$, which was greater than the average spontaneous twitch frequency of the tissues. The width of the tissue was measured during image analysis, and the thickness was conservatively estimated be to $500 \mu \mathrm{m}$ (based on measurements of EHTs from histology). The cross-sectional area was calculated assuming an elliptical cross section ( $A=\pi / 4 \times$ width $\times$ thickness), and used to normalize force into stress for each tissue.

\subsection{Measurements of calcium dynamics}

Intracellular $\mathrm{Ca}^{2+}$-transients were analyzed using Fluo-4, under electrical stimulation at $1.5 \mathrm{~Hz}$ in a Tyrode buffer with $1.8 \mathrm{mM} \mathrm{Ca}^{2+}$ at $37^{\circ} \mathrm{C}$. After 3 weeks in culture, the braces were removed and EHTs were allowed to contract against the reduced load for $24 \mathrm{~h}$ before recording the $\mathrm{Ca}^{2+}$-transients on the unstiffened, $\mathrm{K}_{1}$, posts. For Fluo loading, the EHTs were incubated in EHT media containing $5 \mu \mathrm{M}$ Fluo-4, AM (Invitrogen $\mathrm{F} 14201$ ) for $1 \mathrm{~h}$ at $37^{\circ} \mathrm{C}$, followed by an additional $30 \mathrm{~min}$ incubation at $37^{\circ} \mathrm{C}$ in EHT media. Videos of at least 5 beats were recorded at 66.7 FPS on a Nikon Ti-E epi-fluorescent microscope, with a $2 \times$-objective (which allowed visualization of whole EHTs) and 0.7 coupler between the microscope and Hamamatsu flash V3 camera. The average Fluo intensity over a region of interest containing an area of the tissue between the posts $\left(\mathrm{ROI}_{\text {Fluo }}\right.$ ) was tracked using a custom Matlab program. To account for EHT shortening during contractions, the edges of the $\mathrm{ROI}_{\text {Fluo }}$ were adjusted at each frame to be $1.25 \mathrm{~mm}$ in distance from the centroids of the posts and followed the contour of the lateral edges of the tissue. Traces of the average Fluo intensity within the $\mathrm{ROI}_{\text {Fluo }}(\mathrm{F})$ over time were then used to calculate the calcium kinetics for the EHTs. The relaxation time constant, $\tau$, was determined from fitting an exponential curve of the form $\mathrm{F}(\mathrm{t})=\mathrm{A} e^{-\mathrm{t} / \tau} \pm \mathrm{B}$, to the decay phase of the Fluo transient profile, where $t$ is time in seconds and $\mathrm{A}$ and $\mathrm{B}$ are fitted constants.

\subsection{Isometric force measurements}

Isometric force versus tissue length relations were obtained following a similar procedure as previously described [15,23]. Briefly, EHTs were manually removed from the posts and immediately transferred into a bath of Tyrode buffer with $1.8 \mathrm{mM} \mathrm{Ca}^{2+}$. A gravity fed perfusion system maintained the bath temperature at $37^{\circ} \mathrm{C}$. EHTs were secured to modified 22-g needles extending from the lever arms of a force transducer (Cambridge Technology, Inc., model 400A) and length-controlling motor (Cambridge Technology, Inc., Model 400A) by looping and tightening sutures around the needles and the tissue ends. Slack length was determined using a stereomicroscope with a reticle in the eyepiece to optically match the width of the tissue before and after attachment to the force transducer and motor lever arms. By adjusting the motor arm position, tissue length was slowly increased (in approximately $0.08 \mathrm{~mm}$ increments, which was equivalent to about $2.5 \%$ strain increments) while twitch and passive forces were continuously recorded. For the experiments shown, EHTs were all electrically paced via field stimulation at $1.75 \mathrm{~Hz}$, consistent with the in situ measurements. Tissues were stretched until a peak in twitch amplitude was achieved (Fig. S3 A). Force and length were recorded via custom LabView software and subsequently analyzed with a custom Matlab program, to obtain passive tension and isometric twitch force (average of at least 5 sequential twitches). Stress was calculated using the same cross-sectional area as for the in situ measurements.

\subsection{Gene expression analysis}

EHTs were dissociated for gene expression analysis 1 day after the 3- 
week in situ contractile analysis was performed. Total cellular RNA was extracted using RNeasy Plus Micro Kit (QIAGEN, 74034) according to the manufacturer's instructions except for the following modifications. Individual tissues were pre-digested using $2 \mathrm{mg} / \mathrm{mL}$ Proteinase $\mathrm{K}$ in $100 \mu \mathrm{L}$ DPBS for $10 \mathrm{~min}$ at $56^{\circ} \mathrm{C}$ in agitation. Cells were then lysed by adding $350 \mu \mathrm{L}$ of RLT Plus Buffer (supplemented with 1\% 2-mercaptoethanol), and cleared through the gDNA Eliminator Mini Spin Column. The RNA lysate was finally prepared for binding to the RNeasy MinElute Spin Column by adding $250 \mu \mathrm{L}$ of 200 proof ethanol. All subsequent steps were performed according to the supplier's recommendations. $10 \mu \mathrm{L}$ of eluted RNA (corresponding to 75-150 ng) were subjected to reverse transcription using M-MLV RT (Invitrogen, 28025013) according to the manufacturer's protocol and including RNase OUT (Invitrogen, 10777019). Quantitative real-time reverse transcription PCR (RT-qPCR) was performed with SYBR Select Master Mix (Applied Biosystems, 4472913) using $2 \mathrm{ng}$ of cDNA and $400 \mathrm{nM}$ forward and reverse primers. Reactions were run on a 7900HT Fast Real-Time PCR System (Applied Biosystem, 4329001), and data was analyzed using the $\triangle \triangle \mathrm{Ct}$ method using HPRT1 as the housekeeping gene. Primers were designed using PrimerBlast, and confirmed to amplify a single product. A complete list can be found in the Supplemental information.

For comparison with EHT gene expression, the cDNA of fetal left ventricles obtained from a previously published study [31] was reanalyzed here for the genes of interest. The human fetal hearts (gestational age 91-105) were originally obtained from the University of Washington Birth Defects Laboratory under a program supported by the National Institutes of Health.

\subsection{Western blot}

Protein lysates were obtained using ice-cold $1 \times$ RIPA buffer containing protease and phosphatase inhibitors (Cell Signaling 9806), and freshly supplemented with $1 \mathrm{mM}$ phenylmethylsulfonyl fluoride (PMSF). Individual EHTs were homogenized in $400 \mu \mathrm{L}$ of buffer using a TissueRuptor II equipped with disposable probes (QIAGEN 9002755 and 990890), all performed on ice. The lysate was clarified from insoluble material by centrifugation at $16,000 \mathrm{~g}$ for $10 \mathrm{~min}$ at $4^{\circ} \mathrm{C}$. The protein concentration was assessed using the Pierce BCA Protein Assay Kit (ThermoFisher, 23225) according to the manufacturer's instructions. After addition of Laemmli Sample Buffer (Bio-Rad, 1610747) to a final concentration of $1 \times$, and 2-mercaptoethanol to a final concentration of $2.5 \%$, the samples were denatured by heating at $95^{\circ} \mathrm{C}$ for 5 min.

For electrophoretic separation, $5 \mu \mathrm{g}$ of protein for each sample was loaded onto 4-20\% Mini-PROTEAN TGX Precast Protein Gels (Bio-Rad 4561094) and run at $100 \mathrm{~V}$ for $90 \mathrm{~min}$ using $1 \times$ Tris/Glycine/SDS running buffer (Bio-Rad, Cat. \#1610732). Proteins were transferred onto Immobilion-P PVDF membranes (Millipore Sigma IPVH00010) by means of tank blotting in $1 \times$ Tris/Glycine (Bio-Rad 1610734) supplemented with $20 \%$ methanol; transfer was performed at $100 \mathrm{~V}$ for $90 \mathrm{~min}$ at $4{ }^{\circ} \mathrm{C}$. Membranes were blocked in PBS supplemented with $0.1 \%$ Tween-20 (hereafter PBST) and 5\% bovine serum albumin (BSA) for $1 \mathrm{~h}$ at room temperature. Primary antibody incubation was performed overnight at $4{ }^{\circ} \mathrm{C}$ under agitation, and antibodies were diluted in the same blocking buffer. The following antibodies were used: mouse monoclonal anti-MYH7 (clone A4.95, Developmental Studies Hybridoma Bank), 1:1000 dilution; mouse monoclonal anti-MYH6 (clone BA-G5, Abcam ab50967), 1:1000 dilution; goat polyclonal antiLamin A/C (Santa Cruz sc-6215), 1:500 dilution. Membranes were washed three times in PBST for $10 \mathrm{~min}$ at room temperature, incubated for $1 \mathrm{~h}$ at room temperature with species-appropriate HRP-conjugated secondary antibodies (ThermoFisher 31430 and A27014; diluted $1: 10,000$ in blocking buffer), and washed three times in PBST for $10 \mathrm{~min}$ at room temperature. Chemiluminescent reaction was initiated by incubation with SuperSignal West Femto Maximum Sensitivity
Substrate (ThermoFisher 34095), and images were acquired using a ChemiDoc Imaging System (Bio-Rad) in "high resolution" mode. Before re-probing for the housekeeping protein (lamin A/C), membranes were treated with Restore Plus western blot stripping buffer (ThermoFisher 46430), washed three times, and re-blocked. Given the large number of samples to be quantitatively compared, we ran multiple gels that included one common sample, which was used as internal normalizer to compare protein abundance across gels. Densitometric quantification of Western blots was performed using ImageJ, and protein estimation was normalized on the levels of lamin A/C within each lysate. This strategy allows to correct for the approximate number of nuclei (and thus cells) within each EHT [32].

\subsection{Immunofluorescence and image analysis}

EHTs were submerged in $140 \mathrm{mM} \mathrm{KCl}$ for $30 \mathrm{~s}$ to induce relaxation, then fixed with $4 \%$ paraformaldehyde in PBS for $15 \mathrm{~min}$, washed once in PBS, and dehydrated in $30 \%$ sucrose in deionized water at $4{ }^{\circ} \mathrm{C}$ overnight. The following day, EHTs were removed from the tips of the PDMS posts, embedded and frozen into blocks of Tissue-Plus ${ }^{\circledR}$ O.C.T. compound (Scigen Scientific, 4583). For histology, $5 \mu \mathrm{m}$ thick sections were obtained using a Leica CM1850 cryostat. Slides were blocked with $10 \%$ goat serum for $1 \mathrm{~h}$ at room temperature, followed by overnight incubation at $4{ }^{\circ} \mathrm{C}$ with mouse monoclonal $\alpha$-actinin primary (mouse monoclonal antibody, Sigma-Aldrich A7811) at 1:800. The next day, samples were incubated with AlexaFluor-594-conjugated goat antimouse at 1:200, AlexaFluor-488-Phalloidin at 1:250 (LifeTechnologies A12379), and Hoechst at 1:500 (Invitrogen H1399) together for $1 \mathrm{~h}$ at room temperature. Cover slides were mounted with Fluoromount- $\mathrm{G}^{\mathrm{TM}}$ (ThermoFisher). Images were taken on either a Nikon TEi epi-fluorescent microscope or a Nikon A1 confocal microscope. Resting sarcomere length was calculated using Cytospectre [33]. The Z-disk width was calculated using a custom Matlab program that calculated the major axis length of the objects from thresholded images stained for $\alpha$ actinin. Cell area was calculated by manually outlining the $\alpha$-actinin positive regions and counting the number of enclosed nuclei. The average cell area reported for each tissue is the sum of all measured $\alpha$ actinin positive area divided by the total nuclei (imaged area included at least 50 nuclei for each tissue).

\subsection{Transmission electron microscopy (TEM)}

EHT samples were kept on the posts with braces and fixed overnight in $4 \%$ glutaraldehyde in $0.1 \mathrm{M}$ sodium cacodylate buffer at room temperature, then stored for $1-3$ days at $4{ }^{\circ} \mathrm{C}$. Subsequently, fixed tissues were washed in buffer, then treated with $2 \%$ buffered osmium tetroxide for $1 \mathrm{~h}$ on ice. Tissues were then washed in water at room temperature and dehydrated through a graded series of ethanol on ice, followed by two changes of propylene oxide at room temperature. Each tissue was infiltrated in a 1:1 mixture of propylene oxide and Epon Araldite epoxy resin overnight at room temperature. The next day the tissues were infiltrated with two changes of Epon Araldite, placed in flat embedding molds and polymerized overnight at $60{ }^{\circ} \mathrm{C}$. Thin sections $(80 \mathrm{~nm})$ were taken and the sections were imaged using a JEOL 1230 TEM.

\subsection{Biological replicates and statistical analysis}

All values are reported as mean \pm standard error of the mean (S.E.M). Separate experiments were run on EHTs cast from independent differentiations, and a final experiment was performed by pooling hiPSC-CMs from three separate differentiations, all showing consistent trends in auxotonic twitch force between the different afterload groups (Fig. S2). Unless otherwise indicated, all images and reported data are for a batch of EHTs containing hiPSC-CMs pooled from 3 independent differentiations of hiPSC at different passages. All data points shown in figures represent values for a single tissue: 15 tissues per group for in 

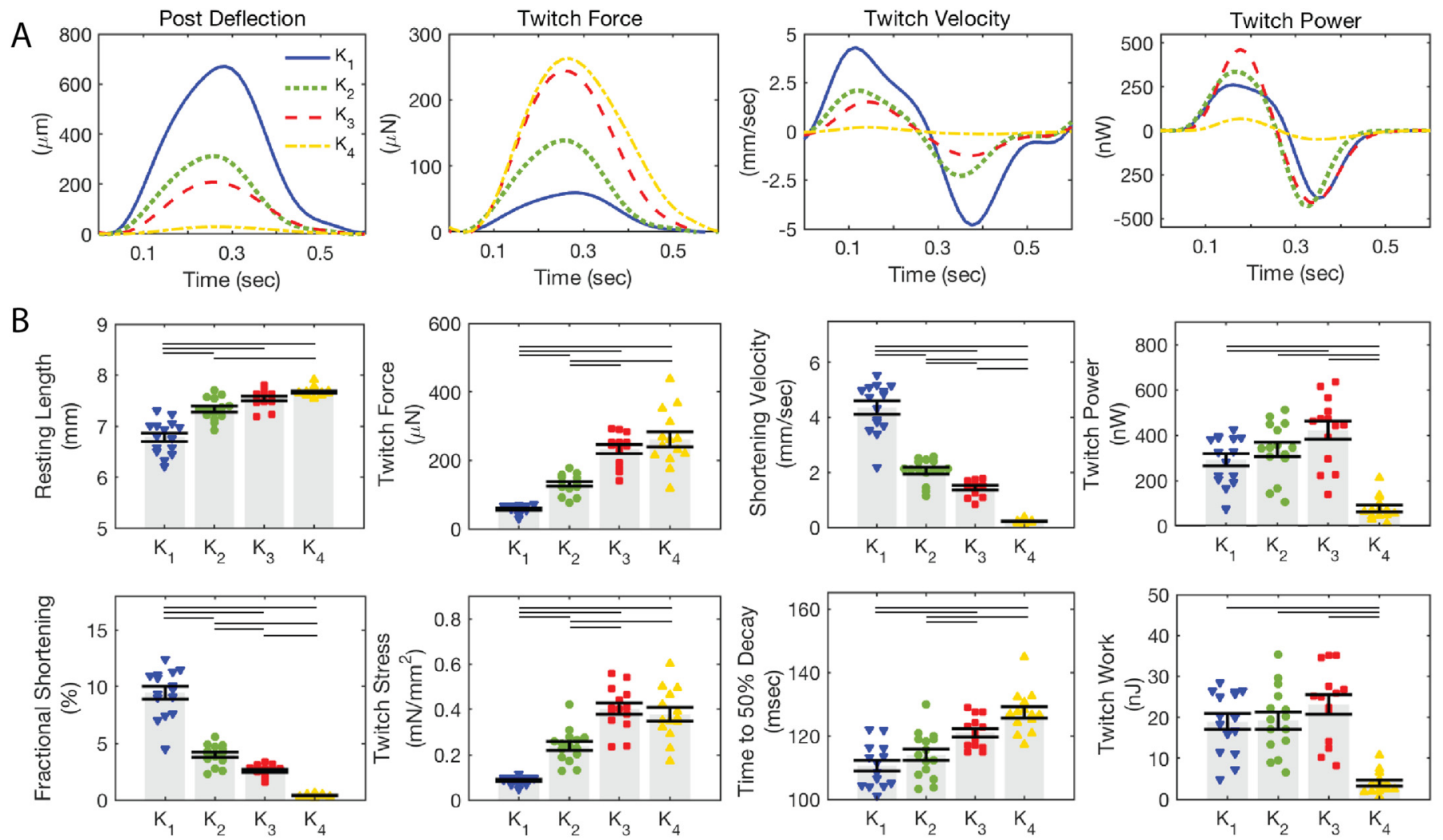

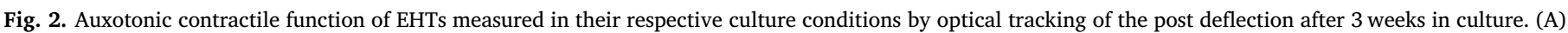

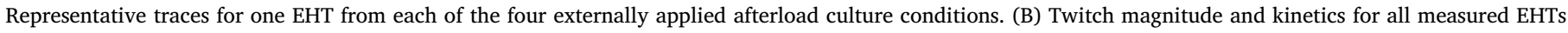

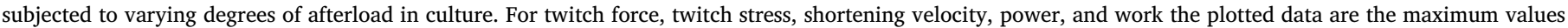

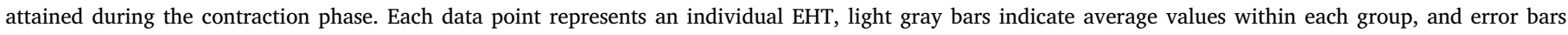
represent S.E.M. Horizontal bars between groups indicate statistical significance.

situ optical tracking, 3 to 6 per group for isometric force measurements, 4 to 5 per group for RT-qPCR, 3 to 6 per group for calcium imaging, 3 to 6 per group for western blots, and 2 to 6 per group for histology. Comparison of results between the 4 different non-zero afterload culture conditions was done with a one-way ANOVA and Tukey-Kramer (HSD) post-hoc test. Differences at a p-value $<0.05$ were considered statistically significant.

\section{Results}

\subsection{Adjustable afterload platform}

To examine the effect of afterload on hiPSC-CM maturation, we modified previously established techniques $[29,30]$ to generate 3D EHTs by casting hiPSC-CMs in a fibrin gel between two posts, one rigid and one flexible (bending stiffness $\mathrm{K}_{1}$ ). The day after EHT casting, externally applied afterload was either removed by detaching one end of the EHT from the flexible post (denoted UL, for unloaded) or increased by employing braces that magnetically clamped around a pair of posts, effectively shortening the post lengths and increasing the resistance to contraction (Fig. 1A-B). The braces were 3D printed from PLA, a biocompatible material for cardiomyocyte cultures [34]. This platform allows for adjustable afterload conditions and was implemented for four distinct afterloads: one low $\left(\mathrm{K}_{1}=0.09 \mu \mathrm{N} / \mu \mathrm{m}\right)$, two moderate $\left(\mathrm{K}_{2}=0.45 \mu \mathrm{N} / \mu \mathrm{m}\right.$ and $\left.\mathrm{K}_{3}=1.2 \mu \mathrm{N} / \mu \mathrm{m}\right)$, and one nearly isometric or high $\left(\mathrm{K}_{4}=9.2 \mu \mathrm{N} / \mu \mathrm{m}\right)$ condition. The brace and post lengths used to produce each afterload condition are shown in Fig. 1A-D. All EHTs compacted and displayed synchronous spontaneous contractions within the first week of culture. Substantial increases in contractile force were observed for the next two weeks, and after three weeks in culture, all
EHTs subjected to external afterload deformed the flexible post a measurable amount (Fig. 1E). While EHTs can be maintained up to several months on this platform, we observed minimal or no increase in EHT fractional shortening for culture beyond three weeks. Compared to previous platforms that studied afterload [21-23], this approach provides a substantially wider range of afterload conditions and is more readily adaptable in its implementation.

\subsection{Auxotonic contractile performance of EHTs under different afterloads}

In vivo, increases in afterload result in reduced fractional shortening of cardiomyocytes, decreased shortening velocity, and greater contractile force to preserve cardiac output [35]. This phenomenon demonstrates the force-velocity relationship of muscle, and has been observed [36] and modeled [37] in the literature for auxotonic contractions of the whole heart. Our afterload platform allows for in situ assessment of EHT performance under variable contractile resistance, which has more recently been shown to also affect the force [21] and power [38] of contraction of neonatal rat cardiomyocytes. After three weeks in culture, the auxotonic contractile function of EHTs was measured in situ via optical tracking of the flexible post deflection and subsequent analysis of the force profiles (Fig. 2). A representative example of an unloaded EHT, and EHTs contracting on the four tested posts stiffness $\left(\mathrm{K}_{1}\right.$ through $\left.\mathrm{K}_{4}\right)$ is shown in the Movie S1. The forces of the unloaded tissues could not be measured in situ since they were not attached to a flexible post. For the four discrete afterload conditions, we observed that the resting length of the EHTs correlated positively with the bending stiffness of the posts, while the fractional shortening correlated negatively. Twitch force generated by the EHTs increased with bending stiffness, while shortening velocities and twitch relaxation 
rates decreased. Specifically, the twitch force generated by the EHTs significantly increased from $57 \pm 3 \mu \mathrm{N}$ on $\mathrm{K}_{1}$, to $131 \pm 7 \mu \mathrm{N}$ on $\mathrm{K}_{2}$, and $233 \pm 12 \mu \mathrm{N}$ on $\mathrm{K}_{3}$, with a modest increase to $262 \pm 22 \mu \mathrm{N}$ on $\mathrm{K}_{4}$. The larger variability in twitch force observed for the $\mathrm{K}_{4}$ condition can be attributed to the small deflection of the post, which yielded 3 to 4 pixels of movement $\left(76 \mu \mathrm{N} /\right.$ pixel for $\left.\mathrm{K}_{4}\right)$, as opposed to biological variability in EHT performance. The average cross-sectional area of all the EHTs cultured on the posts was $0.64 \mathrm{~mm}^{2} \pm 0.03 \mathrm{~mm}^{2}$. While there was some variability in width of the tissues between groups, it did not correlate with increasing afterload conditions. Hence, when the twitch force of each tissue was normalized by its cross-sectional area, the twitch stress showed the same trend as twitch force. Because shortening velocity during a twitch correlated inversely with post stiffness, twitch power and work showed maximum values at moderate afterload $\left(\mathrm{K}_{3}\right)$, and minimum values at the nearly isometric afterload $\left(\mathrm{K}_{4}\right)$. Overall, we find that EHTs exhibit a similar contractile response to increasing afterload as has been observed in vivo, exhibiting an inverse relationship between twitch force and shortening velocity that demonstrates agreement with the force-velocity relationship of adult striated muscle. We note that EHTs performed best (maximized power) under the moderate afterload condition $\left(\mathrm{K}_{3}\right)$.

\subsection{Isometric contractile function and Frank-Starling response of EHTs}

Cardiac muscle, like skeletal muscle, exhibits an inverse relationship between force of contraction and shortening velocity, with maximal velocity achieved at unloaded conditions and maximal force produced under isometric conditions [36,39]. Since the level of afterload directly affects the fractional shortening and shortening velocity [39], it is necessary to measure all EHTs under the same loading condition to accurately compare their maximal contractile capacity (inotropy). Therefore, we measured the EHTs that had been cultured with different afterload conditions under isometric loading by removing them from the posts and affixing them to a force transducer system (Fig. 3A). All
EHTs showed a positive Frank-Starling response, i.e. stretch-induced augmentation of twitch force [40], when they were stretched longitudinally (Fig. 3B and Fig. S3A). A representative isometric twitch force versus strain curve of an EHT from each afterload culture group is shown in Fig. 3C. EHTs showed a peak isometric twitch force at $\mathrm{L}_{\max }$, which occurred near $75 \%$ strain on $\mathrm{K}_{2}, \mathrm{~K}_{3}$, and $\mathrm{K}_{4}$ (Fig. 3C). The active force generating capacity of each tissue was quantified in terms of both the slope of the Frank-Starling curve (Fig. 3D), and maximum observed twitch force at $\mathrm{L}_{\max }$ (Fig. 3E). EHTs cultured without external afterload (UL) developed $139 \pm 42 \mu \mathrm{N}$ of maximum twitch force at $\mathrm{L}_{\max }$, which was significantly less than those subjected to external afterload in culture. EHTs subjected to the low afterload $\left(\mathrm{K}_{1}\right)$ developed $425 \pm 36 \mu \mathrm{N}$ of maximum twitch force at $\mathrm{L}_{\max }$, while those cultured under high afterload $\left(\mathrm{K}_{4}\right)$ achieved a significantly higher maximum twitch force of $656 \pm 14 \mu \mathrm{N}$. Both the maximal twitch force and slope of the Frank-Starling curves for the moderate afterload groups $\left(\mathrm{K}_{2}\right.$ and $\mathrm{K}_{3}$ ) were similar to those in the high afterload group $\left(\mathrm{K}_{4}\right)$. The rate of force development during an isometric twitch also increased with afterload up to the moderate, $\mathrm{K}_{2}$, culture condition but was not further increased with very high afterload in culture (Fig. $3 \mathrm{~F}$ and Fig. S3B). However, there was no observed difference in time to peak or time to $50 \%$ amplitude decay between the four afterload conditions. This finding indicates that EHTs subjected to afterload obtain contraction and relaxation properties that are conducive to proper performance of systolic and diastolic ventricular wall function in the heart. As observed by others [41], the EHTs within each group generated higher twitch forces under isometric conditions compared to auxotonic contractions. Nevertheless, comparing the auxotonic and isometric performance for each tissue confirmed that these values correlated with each other (Fig. S4). Together, the auxotonic and isometric contractile assessment indicate that there are functional benefits of culturing EHTs with increasing afterload. However, the improvements in isometric contractile performance appear to saturate at moderate afterloads $\left(\mathrm{K}_{2}\right)$.
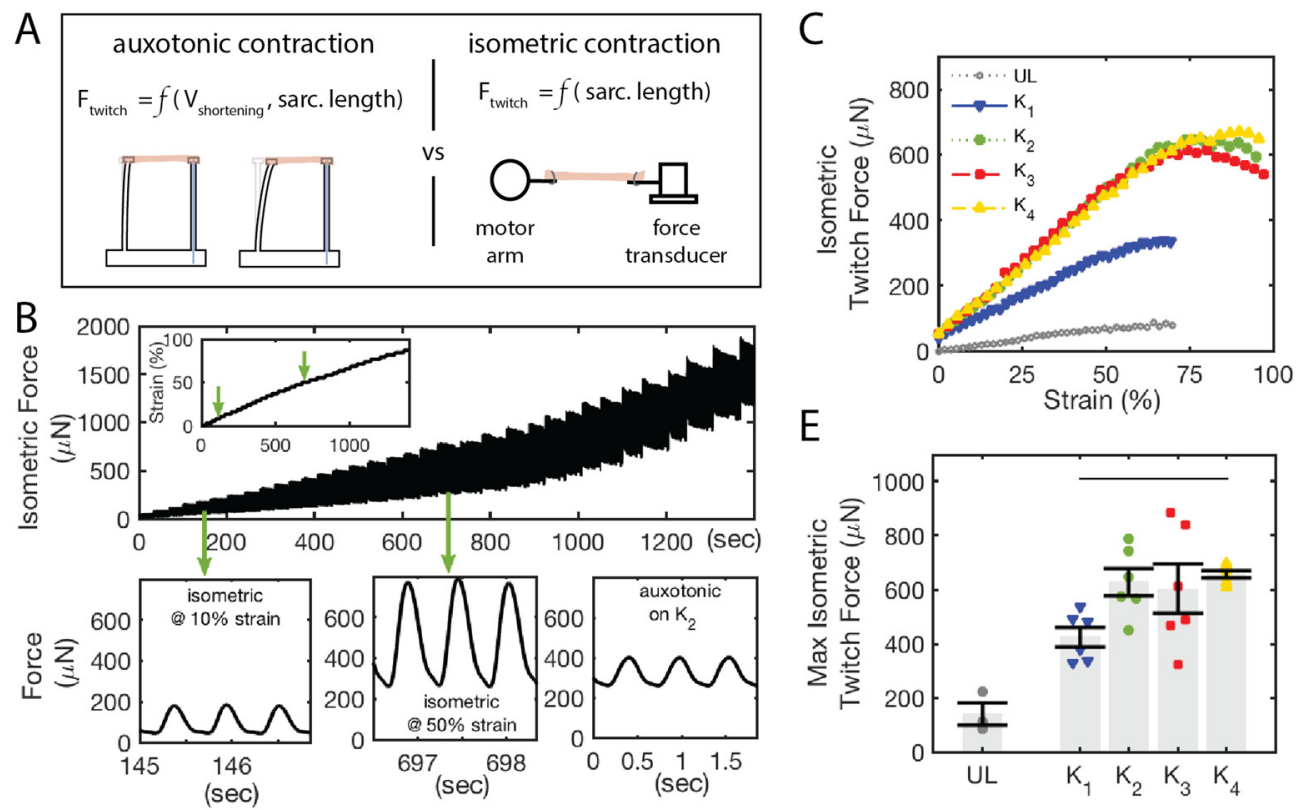

E

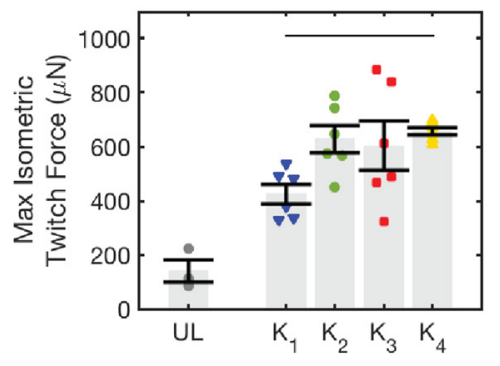

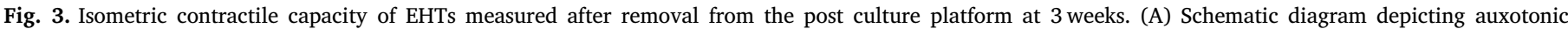

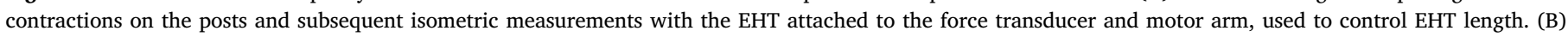

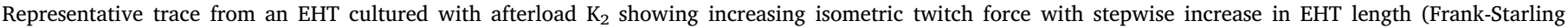

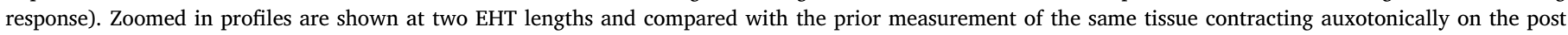

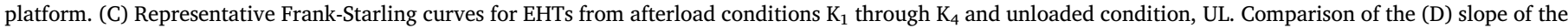

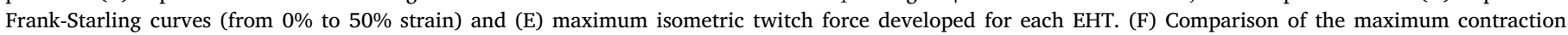

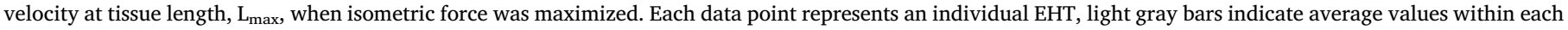
group, and error bars represent S.E.M. Horizontal bars between groups indicate statistical significance between the $\mathrm{K}_{1}$ through $\mathrm{K}_{4}$ groups. 
A
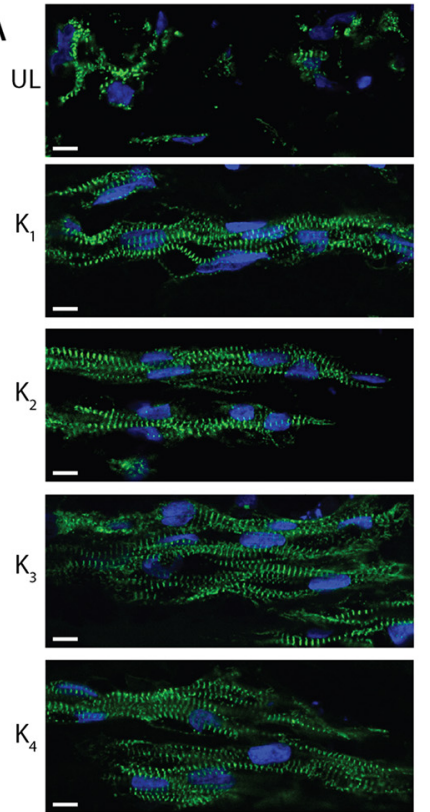

B
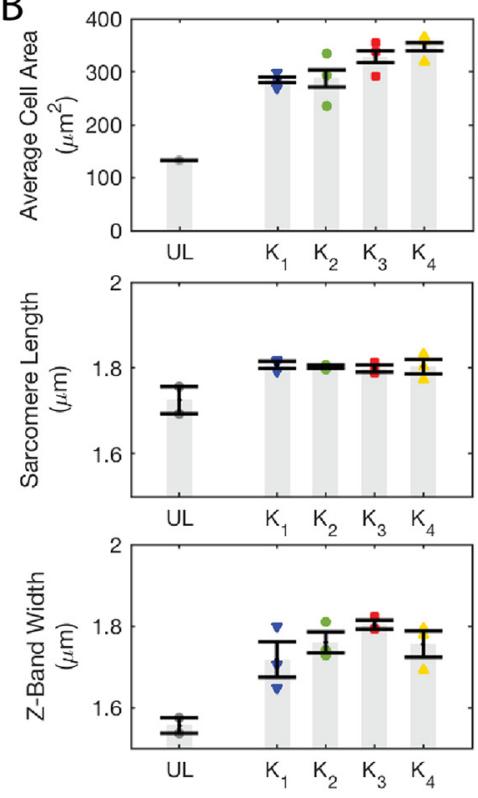
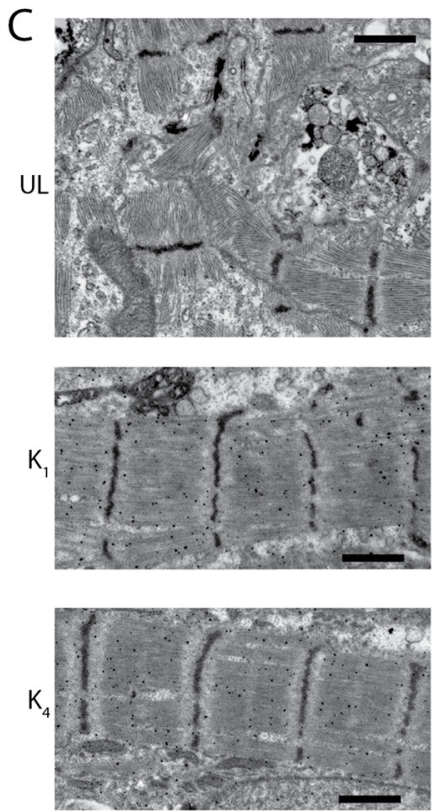

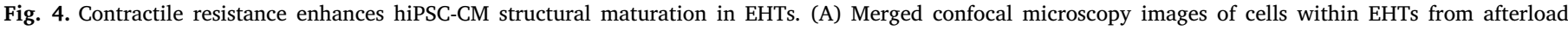

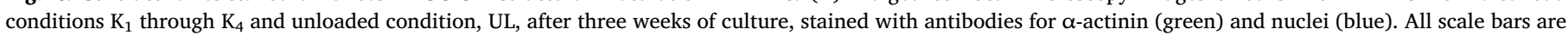

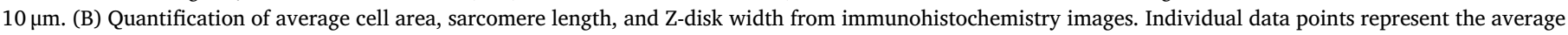

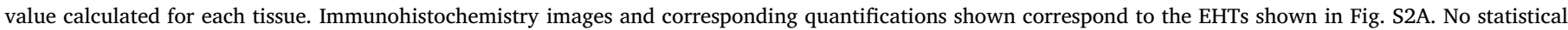

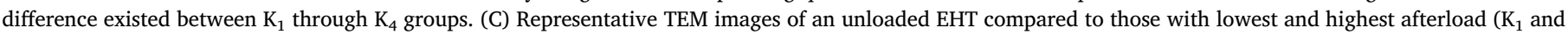
$\mathrm{K}_{4}$ ). All scale bars are $1 \mu \mathrm{m}$. (For interpretation of the references to colour in this figure legend, the reader is referred to the web version of this article.)

\subsection{Afterload enhances structural maturation}

We investigated whether the observed increase in contractile function from afterload corresponds to improvements in the underlying hiPSC-CM sarcomere structure associated with cardiac maturation. Structurally, as expected, all EHTs subjected to afterload had notable alignment of cells in the longitudinal direction, while those cultured in the absence of external afterload (UL) had cells that were rounded and lacked any apparent orientation (Fig. 4A and Fig. S5). Furthermore, we found that all afterload conditions resulted in a larger hiPSC-CMs when compared to unloaded EHTs, but we did not observe a significant increase in cell size when comparing amongst afterload conditions, assessed either longitudinally (Fig. 4B) or cross-sectionally (Fig. S6). Immunohistochemistry staining for the $Z$-disk protein $\alpha$-actinin revealed a similar trend in resting sarcomere length and Z-disk width (indicative of myofibril alignment/bundling), with improvement in hiPSC-CMs in EHTs subjected to afterload $\left(\mathrm{K}_{1}\right.$ through $\mathrm{K}_{4}$ ) compared to the unloaded group (UL). Using TEM, we examined the ultrastructure of EHTs subjected to the highest and lowest afterload conditions and those in the unloaded condition (Fig. 4C). We observed cardiomyocytes of EHTs in the unloaded condition had sarcomeres that were disorganized and less developed. For the afterload conditions, we observed well-organized sarcomeres that had overlapping thick and thin filaments and distinct Z-bands and A-zones. Together, we find that afterload conditions have improved cell size and sarcomere structure when compared to unloaded EHTs, but improvements between the different conditions of afterload were minimal.

\subsection{Calcium transient kinetics improve with increasing afterload}

We investigated if the improvements in contractile function correlated with improved calcium handling. The calcium handling apparatus and excitation contraction coupling in hiPSC-CMs is known to be underdeveloped, and calcium dynamics are slower than in adult cardiomyocytes [2]. We utilized Fluo-4 to image $\mathrm{Ca}^{2+}$ transients in the EHTs (Fig. 5A), and focused our analysis on the kinetics of the $\mathrm{Ca}^{2+}$ transients (Fig. 5B). We observed a significant improvement in the time to peak, time to $50 \%$ decay, and relaxation time constant, $\tau$, with increasing afterload (Fig. 5C). While improvements in inotropy resulting from applied afterload in culture appear to saturate at moderate afterload (Fig. 3), our results with Fluo-4 indicate that at least some aspects of the calcium handling are continually improved with applied afterload in culture.

\subsection{Cardiac specific gene and protein expression improve with afterload}

Next, we investigated whether the phenotypic changes observed with increased afterload correlated with gene expression changes and saw evidence for maturation with increasing afterload (Fig. 6A). In human ventricular muscle, the $\beta$-myosin heavy chain subunit ( $\beta M H C$, encoded by $M Y H 7$ ) is predominant compared with the $\alpha$-heavy chain subunit ( $\alpha$ MHC, encoded by MYH6), and increases in the ratio of $\beta M H C$ to $\alpha \mathrm{MHC}$ are associated with hiPSC-CM maturation [2]. Quantitative RT-PCR data showed a decreasing MYH6 and increasing MYH7 expression with increasing afterload (Fig. 6A). Notably, for the highest afterload condition $\left(\mathrm{K}_{4}\right.$ ), the expression of $M Y H 6$ and $M Y H 7$ (relative to HPRT) were approaching fetal levels after the three-week culture period (Fig. 6B), but still far from reported values for adult myocardium [42]. Confirming these findings, the protein expression of $\beta \mathrm{MHC}$ and $\mathrm{\alpha MHC}$ showed similar trends to the gene expression data (Fig. S7), and the ratio of $\beta M H C$ to $\alpha \mathrm{MHC}$ protein expression was significantly increased between the $\mathrm{K}_{1}$ and $\mathrm{K}_{4}$ groups (Fig. $6 \mathrm{C}$ ). We also observed that increasing afterload led to increasing gene expression of myosin light chain 2 (MYL2), cardiac actin (ACTC1), and cardiac troponin I (TNNI3), all markers of cardiac maturation and key proteins involved in sarcomere assembly and muscle contraction (Fig. 6A). The gene expression of melusin (ITGB1BP2), a mechanosensitve muscle-specific chaperone that has protective roles during increased cardiac afterload [43], was also increased in the $\mathrm{K}_{4}$ compared to the $\mathrm{K}_{1}$ and $\mathrm{K}_{2}$ groups.

Having observed more rapid $\mathrm{Ca}^{2+}$ transient kinetics following increased afterload, we also looked at the mRNA expression for proteins associated with different aspects of calcium handling and electrical 
A ROI for $\mathrm{Ca}^{2+}$ imaging

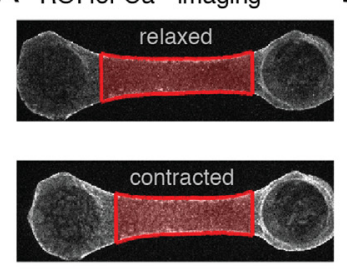

B

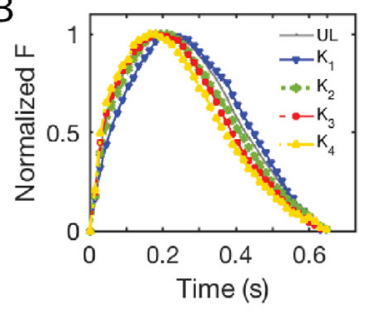

C

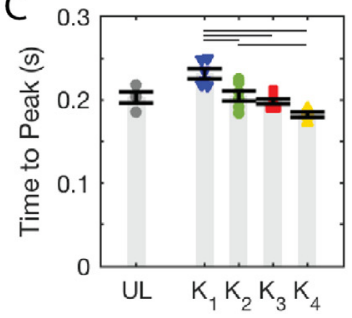

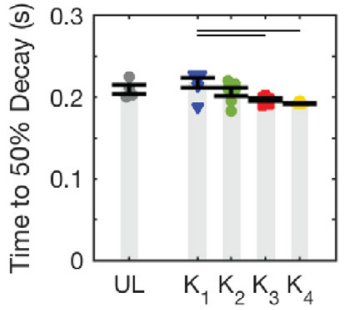

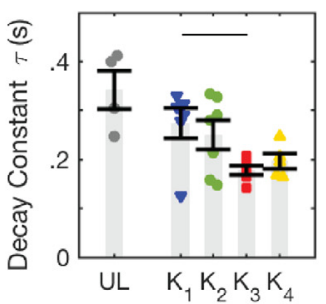

Fig. 5. Calcium kinetics of EHTs improve with increasing afterload in culture. (A) Snapshots from image analysis showing the central region of an EHT used to plot the calcium traces. (B) Representative calcium traces of EHTs from afterload conditions $\mathrm{K}_{1}$ through $\mathrm{K}_{4}$ and unloaded condition, UL. (C) Quantification of the calcium kinetics: time from baseline to peak, time from peak to $50 \%$ amplitude decay, and the fitted value for the exponential decay constant, $\tau$. Each data point represents an individual EHT, light gray bars indicate average values within each group, and error bars represent S.E.M. Horizontal bars between groups indicate statistical significance between the $\mathrm{K}_{1}$ through $\mathrm{K}_{4}$ groups.

excitation (Fig. 6A). We observed a modest increase in the expression of the gene encoding for calsequestrin 2 (CASQ2), and little or no change in those encoding for the 1C subunit of the voltage-dependent L-type $\mathrm{Ca}^{2+}$ channel (CACNA1C) or voltage-gated sodium channel $\alpha$ subunit 5 (SCN5A) as afterload was increased. There also was no difference in RYR2, KCNJ2, and HCN4 ion channels (data not shown). Thus, while there may be other transcription or translational modifications that are affected, it is also possible that improved cardiomyocyte organization and structure is occurring with increased afterload. In summary, we observed increased expression of several key cardiac factors suggesting overall hiPSC-CM maturation improves as external afterload is increased.

\subsection{Gene expression of pathological hypertrophy markers increase with very high afterload}

In vivo, pathologically increased afterload can be modeled with transverse aortic constriction [44], and is known to induce a pathological hypertrophic response with reactivation of the fetal gene program in rodent hearts. A similar gene re-programing typically associated with pathological hypertrophy in humans includes upregulation of atrial natriuretic peptide (NPPA), brain natriuretic peptide (NPPB), $\beta \mathrm{MHC}, \alpha$ skeletal actin (ACTA1), and decreased expression of the endoplasmic reticulum calcium pump SERCA2a (ATP2A2) [25]. Furthermore, pathological hypertrophy is also associated with increased fibrosis and reversion from an fatty acid dominated metabolism at the adult stage to one that is glucose based at the fetal stage [26]. Here, we focused our analysis on a subset of genes associated with pathological hypertrophy, as well as fibrosis markers that others observed to increase with
A
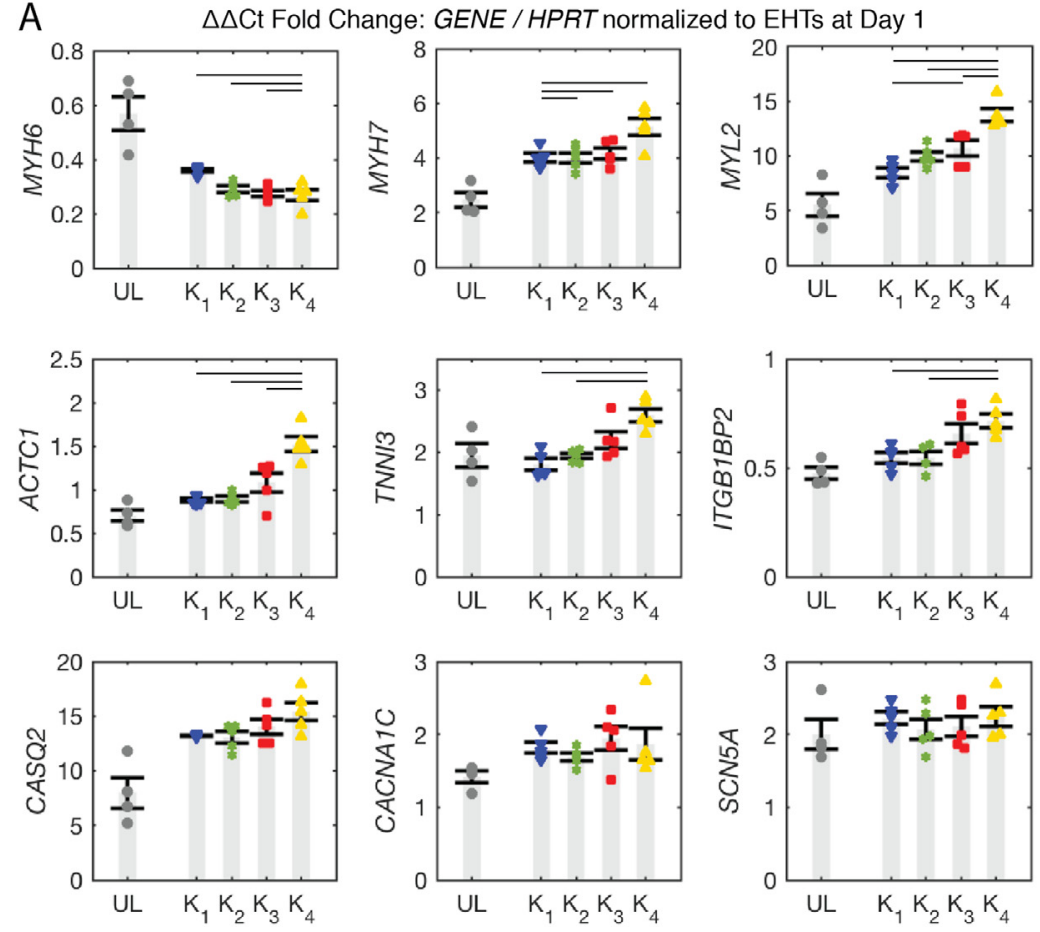

B
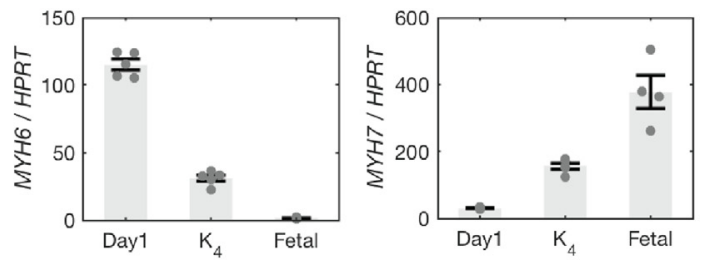

C
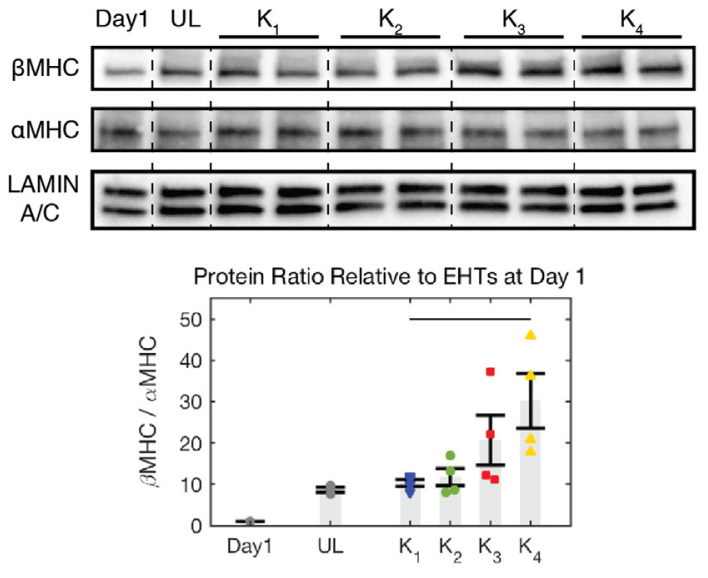

Fig. 6. Cardiac maturation improves with increasing afterload. (A) Changes in cardiac specific gene expression (MYH6, MYH7, MYL2, ACTC1, and TNNI3), the mechanosensitive protein encoded by ITGB1BP2, and markers associated with excitation and calcium handling (CASQ2, CACNA1C, and SCN5A) for EHTs after three weeks with afterload conditions $\mathrm{K}_{1}$ through $\mathrm{K}_{4}$ and unloaded condition, UL. (B) Comparison of $M Y H 6$ and $M Y H 7$ gene expression in EHTs after 1 day and 3 weeks on $\mathrm{K}_{4}$ to that of human fetal ventricles. (C) Western blot of representative samples from each afterload condition for myosin heavy chain- beta ( $\beta \mathrm{MHC}$ ), myosin heavy chain- alpha $(\alpha \mathrm{MHC})$, and lamin A/C proteins. Dashed lines are for visual aide. The corresponding ratio of $\beta$ MHC to $\alpha \mathrm{MHC}$ relative to EHTs after 1 day on afterload conditions $\mathrm{K}_{1}$ through $\mathrm{K}_{4}$ and unloaded condition, UL, is shown below. Each data point represents an individual EHT, light gray bars indicate average values within each group, and error bars represent S.E.M. Horizontal bars between groups indicate statistical significance between the $\mathrm{K}_{1}$ through $\mathrm{K}_{4}$ groups. 
A Pathological Hypertrophy

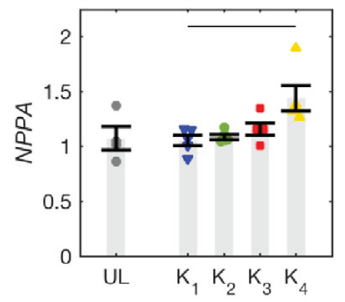

$\triangle \triangle \mathrm{Ct}$ Fold Change: GENE / HPRT normalized to EHTs at Day 1
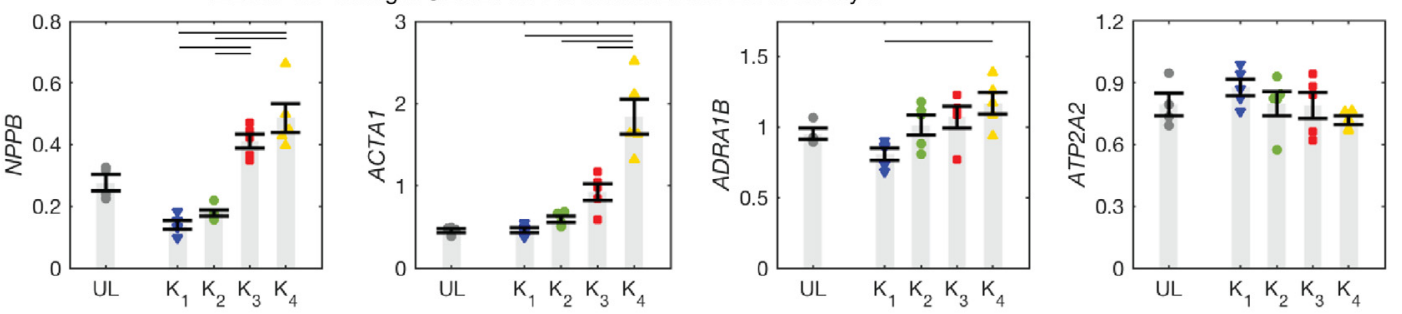

B

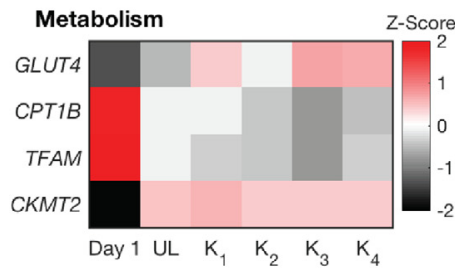

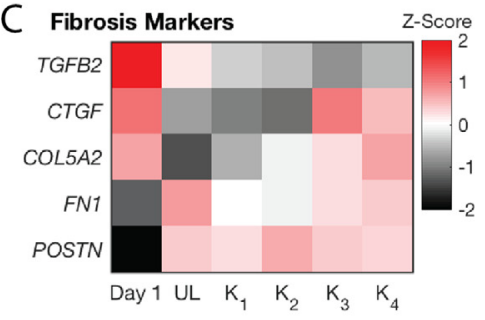

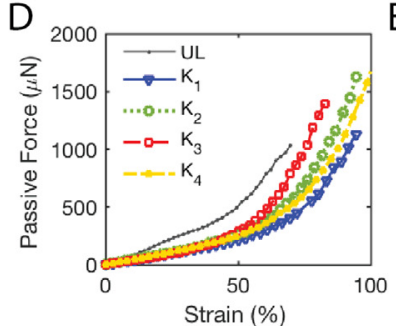

E

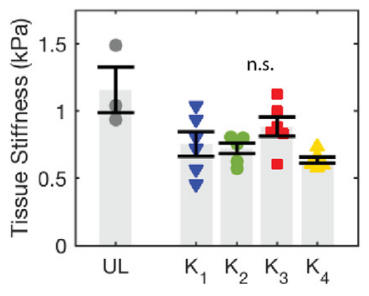

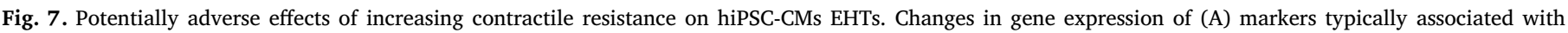

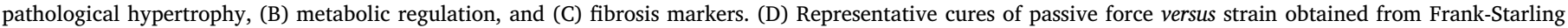

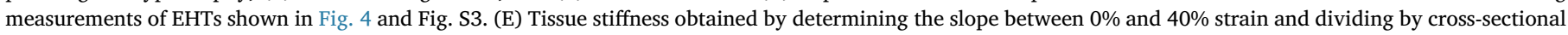

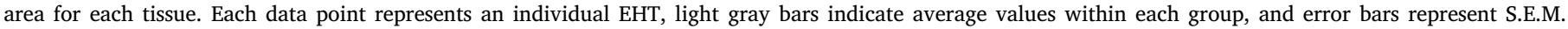
Horizontal bars between groups indicate statistical significance between the $\mathrm{K}_{1}$ through $\mathrm{K}_{4}$ groups.

afterload in vitro when using a similar EHT model composed of neonatal rat cardiomyocytes [22]. Afterload modulated the expression of several of these genes (Fig. 7A). The largest changes were observed for the high afterload condition $\left(\mathrm{K}_{4}\right)$, where NPPA went up by $40 \%$ and ACTA1 went up by $80 \%$ compared to EHTs at Day 1 . Additionally, some aspects of hiPSC-CM metabolism typically associated with a fetal state were increased with external afterload in culture (Fig. 7B and Fig. S8). Specifically, the increasing trend in gene expression of GLUT4 and decreasing trend in $C P T 1 B$ with increasing afterload are indicative of increased glycolysis and decreased fatty acid utilization. This indicates that increasing afterload was ineffective in moving EHTs toward an oxidative metabolism. Finally, expression of some growth factors and extracellular matrix proteins associated with fibrosis were also weakly increasing with afterload (Fig. 7C and Fig. S8).

We then measured the bulk stiffness of the EHTs to determine if the increase in fibrosis markers translated into measurable stiffening at the tissue level. Bulk stiffness of the fibrin gel without cells was approximately $0.1 \mathrm{kPa}$ (data not shown). The addition of hiPSC-CMs and the supporting stromal cells did increase the bulk tissue stiffness over a three week period, but was similar (0.6-0.9 $\mathrm{kPa}$ ) amongst all four nonzero afterload conditions $\left(\mathrm{K}_{1}\right.$ to $\mathrm{K}_{4}$ ) (Fig. 7D-E and S3A). The slight increase in stiffness for the unloaded condition can be attributed to the fact that these tissues were more visibly compacted than EHTs that remained attached to the posts (see Fig. S4 and Movie S1). In all conditions, the matrix stiffness was below the healthy physiological range $(5-15 \mathrm{kPa})$, and far below reported values for fibrotic myocardium $(35-75 \mathrm{kPa})[3,5]$.

In conclusion, our data suggest that a moderate degree of afterload can lead to multiple clear benefits for EHT maturation and function, but that at very high afterload these may be offset by gene expression changes that could prove maladaptive in the long term.

\section{Discussion}

Numerous bioengineering approaches have been proposed to hasten the maturation of hiPSC-CMs in EHTs for drug screening, disease modeling, and therapeutic applications [6,7]. Here, we focused on effects of in vitro afterload, utilizing an adjustable, auxotonic system for EHTs, where we tested a comprehensive range of afterload conditions from unloaded (UL) to a near isometric state $\left(\mathrm{K}_{4}\right)$. When EHTs were measured in situ, increasing afterload resulted in a continuous increase in auxotonic twitch force, and a continuous decrease in fractional shortening, shortening velocity, and relaxation kinetics, whereas twitch work and power were maximized at a moderate $\left(\mathrm{K}_{3}\right)$ afterload. To strictly compare the contractile capacity of EHTs subjected to the different external afterloads in culture, it was necessary to perform twitch force measurements under more standardized, isometric conditions. Our results show that the presence of external contractile resistance is necessary for increased functional maturation. However, the improvement in isometric contractile function can be achieved under moderate loading, $\mathrm{K}_{2}$ in our system. In contrast to the plateau in contractile function, we observed distinct improvements in $\mathrm{Ca}^{2+}$ transient kinetics and in gene expression markers associated with maturation, including isoform switches in myosin heavy chain. At the highest afterload $\left(\mathrm{K}_{4}\right)$, some potentially adverse effects were noted that are discussed below. A summary of the primary responses to afterload is shown in Fig. 8.

Our results agree with previous studies on (mostly neonatal chick and rat) cardiomyocytes in 2D and 3D that show auxotonic twitch force increases as resistance to contraction increases. Studies on single cardiomyocytes have shown that twitch force continuously increases as

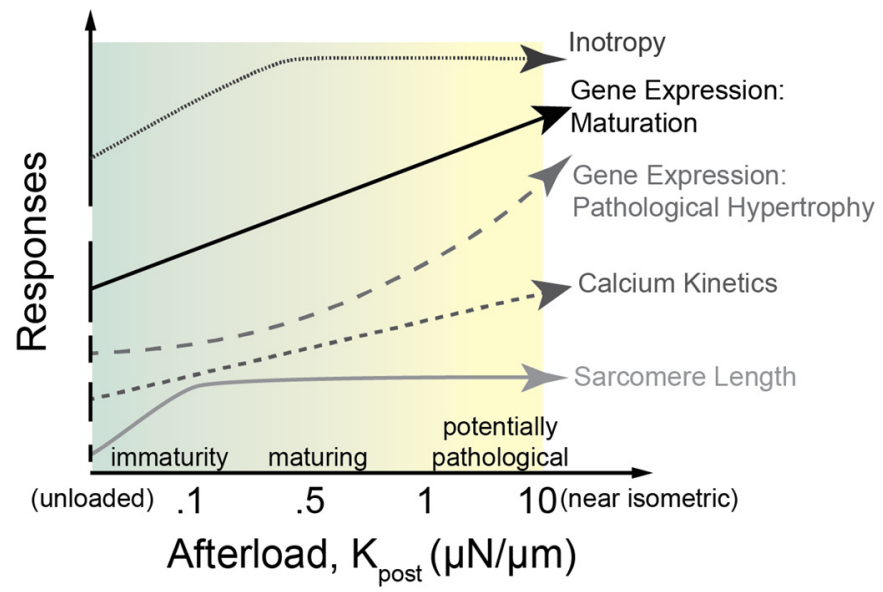

Fig. 8. Summary of measured responses of EHTs subjected to various levels of afterload $\left(\mathrm{K}_{\mathrm{post}}\right)$ for 3 weeks of culture. 
environmental stiffness increases [38,45-50], noting they contract best on physiological $(\sim 10 \mathrm{kPa})$ stiffness substrates [51,52]. With single cells in 2D, environmental stiffness and resistance to contraction are one and the same, and can be more directly compared with native tissue stiffness. However, tissue stiffness and contractile resistance (afterload) are independent parameters for native myocardium, and a 3D EHT platform allows for them to be separately adjusted. Studies varying stiffness in 3D have shown similar results as the 2D counterparts, where increasing matrix stiffness increased twitch force in both neonatal rat based EHTs [21] as well as hiPSC-CM EHTs [53].

So far, studies varying afterload in 3D have been primarily limited to EHTs composed of neonatal rat cardiomyocytes and have shown varied results depending on the level and duration of applied afterload. Boudou et al. [21] tested two discrete post stiffness values, in a 2-fold range (which fall between our $\mathrm{K}_{1}$ and $\mathrm{K}_{2}$ ), and observed increased in situ auxotonic contractile force with increased resistance to contraction. In contrast, Hirt et al. [22] reported a loss of contractile force when EHTs were cultured at high afterloads (similar to our $\mathrm{K}_{4}$ ) for more than a few days, as compared to those cultured at a moderate afterload (with a post stiffness between $K_{2}$ and $K_{3}$ in our system). This is also in contrast to more recent experiments on hiPSC-CM, which reported functional improvement of statically stressed (infinite afterload) EHTs over unloaded ones, when measured under isometric conditions [23]. The discrepancies in auxotonic contractile performance at high/infinite afterload could be a result of differences in the experimental loading regime, measurement techniques, or from differences in the input cardiomyocyte population (neonatal rat cardiomyocytes being more mature than hiPSC-CMs).

Interestingly, none of the 2D or 3D studies followed up their observed correlation between auxotonic twitch performance and afterload with a standardized assessment of contractile capacity (e.g. isometric measurements), which more directly addresses the question of whether applied afterload during culture enhances functional maturation. Without this comparison, it is difficult to determine whether an increase in force due to an increase in afterload derives entirely from an acute biomechanical response based on the force-velocity relationship of muscle, as observed for increasing afterload in vivo [35-37], or whether cells or tissues have increased intrinsic inotropy. Our results indicate that hiPSC-CM EHTs with similar inotropy, such as those cultured on $\mathrm{K}_{2}$ and $\mathrm{K}_{3}$, respond to an increased demand (increase in $\mathrm{K}_{\text {post }}$ ) by contracting more forcefully.

Studies varying preload [12-19], however, often assessed EHTs under isometric conditions, which allows for a comparison of the functional benefits of preload versus afterload. It should first be pointed out that in both the preload and afterload studies (including ours), the two parameters are not fully decoupled. Studies varying stretch, i.e. preload, did not allow for tissue shortening during contractions and therefore also inherently combined preload with infinite afterload. Conversely, when increasing post stiffness, i.e. afterload, we observed increases in resting tension (calculated from $\mathrm{K}_{\text {post }}$ and resting length in Fig. 2B), as did others [21,22]. While preload is often associated with resting tension, in vivo preload is related to the maximum diastolic sarcomere length, which was slightly shorter for our unloaded condition but the same for our $\mathrm{K}_{1}$ through $\mathrm{K}_{4}$ externally applied afterloads (Fig. 4B). Studies varying preload (with infinite afterload) report up to a 2 -fold improvement in contractile force between cyclically stretched tissues and statically stretched controls, for both neonatal $[12,19]$ and hPSC-CM [14] EHTs. The functional improvement of preload is further increased (3-fold) when using unloaded EHTs as a control [14]. Here, we observe a similar (1.5-fold) improvement with applied afterload between groups with the same initial resting sarcomere length $\left(\mathrm{K}_{1}\right.$ to $\mathrm{K}_{4}$ ), and a more dramatic (4.7-fold) improvement when comparing our highest afterload to our unloaded condition. Altogether, this indicates the functional benefits of preload and afterload are similar and potentially additive.

Our results suggest that increasing afterloads promotes maturation of hiPSC-CMs in EHTs, but the improvements in isometric contractile performance appear to saturate at moderate afterloads, $\mathrm{K}_{2}$ in our system. Accordingly, we observed a plateau in certain aspects of structural hiPSC-CM maturation, as sarcomere length and Z-disk width did not increase past the values obtained at low afterload. While the role of increased mechanical loading in mediating a hypertrophic response is well studied [24], we did not observe a significant increase in cell size between our low and high afterload conditions. It is possible the hiPSC-CMs were not given sufficient time in 3D culture to translate gene expression into significant differences in cell size (when quantified by histology). Interestingly, selected cardiac specific gene expression (such as MYH6, MYH7, MYL2, ACTC1, and TNNI3) showed continuous improvements over the range of tested afterloads, suggesting that while the performance of the tissues was not obviously affected, they responded to the increased mechanical loading at the molecular level.

At the highest afterload we also observed some molecular indicators of pathological hypertrophy, fibrosis, and a fetal-like metabolism. Similar findings were reported by Eschenhagen's group with rat cardiomyocytes [22]. One interpretation is that high afterload may induce a pathologic response in hiPSC-CM EHTs. Nevertheless, the interpretation of these results is complicated by the fact that the maturation and pathological responses in hiPSC-CM happened simultaneously in our experiments. Additionally, the increase in fibrosis markers did not manifest as stiffening of the EHT. In the future, it might be possible to more closely mimic the natural sequence of these events by first maturing hiPSC-CM on a moderate afterload condition $\left(\mathrm{K}_{2}\right)$, and then increase the afterload to high levels $\left(\mathrm{K}_{4}\right)$. It might also be required to maintain high afterload over long durations in order for pathological changes to manifest at the tissue level. Overall, we conclude that a moderate level of afterload is the most suited to promote maturation and performance of hiPSC-CM EHTs, while higher level of mechanical loading could be developed into a useful in vitro model of cardiac pathologic remodeling.

\section{Conclusions}

In this study, we highlight the importance of standardized twitch force measurements for accurate evaluation and comparison of contractile capacity, and conclude that increasing afterload is a valid approach to promote functional maturation of EHTs containing hiPSCCMs. These improvements in function correlated with improved hiPSCCM structure and expression of several cardiac genes associated with developmental maturation of the heart. However, when cultured under near isometric conditions, the response showed some features of pathological hypertrophy, with upregulation of fetal gene markers. Overall, allowing EHTs to contract auxotonically under afterload is likely an important part of the maturation puzzle, and should be combined with other established techniques in order to best replicate physiological conditions and enhance maturation for drug screening, disease modeling, and therapeutic applications.

Supplementary data to this article can be found online at https:// doi.org/10.1016/j.yjmcc.2018.03.016.

\section{Funding}

This work was supported in part by the National Science Foundation Grant CBET-1509106 (to N.J.S.); and by NIH Grants R01HL128362, P01HL094374, and a grant from the Fondation Leducq Transatlantic Network of Excellence (to C.E.M.); and by NIH R56AG055594 and R01HL128368 (to M.R.). Trainee support was provided by the NIDH grants F32 HL126332 (to A.L.), T32-HL007312 (to J.D.P.), EMBO LongTerm Fellowship ALTF 488-2017 (to A.B.), and NEI P30 EY001730 (Vision Imaging Core).

\section{Disclosure statement}

None. 


\section{Acknowledgements}

This research was performed with the use of the Cell Analysis Facility Flow Cytometry and Imaging Core in the Department of Immunology and the Garvey Imaging Lab in the Institute for Stem Cell and Regenerative Medicine at the University of Washington. The authors would like to thank Edward Parker at the Vision Imaging Core for his assistance with TEM.

\section{References}

[1] Y. Shi, H. Inoue, J.C. Wu, S. Yamanaka, Induced pluripotent stem cell technology: a decade of progress, Nat. Rev. Drug Discov. 16 (2) (2017) 115-130.

[2] X. Yang, L. Pabon, C.E. Murry, Engineering adolescence: maturation of human pluripotent stem cell-derived cardiomyocytes, Circ. Res. 114 (3) (2014) 511-523.

[3] J.G. Jacot, J.C. Martin, D.L. Hunt, Mechanobiology of cardiomyocyte development, J. Biomech. 43 (1) (2010) 93-98.

[4] P.C. Struijk, V.J. Mathews, T. Loupas, P.A. Stewart, E.B. Clark, E.A. Steegers, J.W. Wladimiroff, Blood pressure estimation in the human fetal descending aorta, Ultrasound Obstetrics Gynecol. 32 (5) (2008) 673-681.

[5] M.F. Berry, A.J. Engler, Y.J. Woo, T.J. Pirolli, L.T. Bish, V. Jayasankar, K.J. Morine, T.J. Gardner, D.E. Discher, H.L. Sweeney, Mesenchymal stem cell injection after myocardial infarction improves myocardial compliance, Am. J. Physiol. Heart Circ. Physiol. 290 (6) (2006) H2196-203.

[6] E. Tzatzalos, O.J. Abilez, P. Shukla, J.C. Wu, Engineered heart tissues and induced pluripotent stem cells: macro- and microstructures for disease modeling, drug screening, and translational studies, Adv. Drug Deliv. Rev. 96 (2016) 234-244.

[7] N.T. Feric, M. Radisic, Maturing human pluripotent stem cell-derived cardiomyocytes in human engineered cardiac tissues, Adv. Drug Deliv. Rev. 96 (2016) $110-134$.

[8] E. Braunwald, R.O. Bonow, Braunwald's Heart Disease : A Textbook of Cardiovascular Medicine, 9th ed., Saunders, Philadelphia, 2012.

[9] A.C. van Spreeuwel, N.A. Bax, A.J. Bastiaens, J. Foolen, S. Loerakker, M. Borochin, D.W. van der Schaft, C.S. Chen, F.P. Baaijens, C.V. Bouten, The influence of matrix (an)isotropy on cardiomyocyte contraction in engineered cardiac microtissues, Integr. Biol. 6 (2014) 422-429, http://dx.doi.org/10.1039/C3IB40219C.

[10] T. Eschenhagen, C. Fink, U. Remmers, H. Scholz, J. Wattchow, J. Weil, W. Zimmermann, H.H. Dohmen, H. Schafer, N. Bishopric, T. Wakatsuki, E.L. Elson, Three-dimensional reconstitution of embryonic cardiomyocytes in a collagen matrix: a new heart muscle model system, FASEB J. 11 (8) (1997) 683-694.

[11] N. Thavandiran, N. Dubois, A. Mikryukov, S. Masse, B. Beca, C.A. Simmons, V.S. Deshpande, J.P. McGarry, C.S. Chen, K. Nanthakumar, G.M. Keller, M. Radisic, P.W. Zandstra, Design and formulation of functional pluripotent stem cell-derived cardiac microtissues, Proc. Natl. Acad. Sci. U. S. A. 110 (49) (2013) E4698-707.

[12] C. Fink, S. Ergun, D. Kralisch, U. Remmers, J. Weil, T. Eschenhagen, Chronic stretch of engineered heart tissue induces hypertrophy and functional improvement, FASEB J. 14 (5) (2000) 669-679.

[13] A. Mihic, J. Li, Y. Miyagi, M. Gagliardi, S.H. Li, J. Zu, R.D. Weisel, G. Keller, R.K. Li, The effect of cyclic stretch on maturation and 3D tissue formation of human embryonic stem cell-derived cardiomyocytes, Biomaterials 35 (9) (2014) 2798-2808.

[14] J.L. Ruan, N.L. Tulloch, M. Saiget, S.L. Paige, M.V. Razumova, M. Regnier, K.C. Tung, G. Keller, L. Pabon, H. Reinecke, C.E. Murry, Mechanical stress promotes maturation of human myocardium from pluripotent stem cell-derived progenitors, Stem Cells 33 (7) (2015) 2148-2157.

[15] N.L. Tulloch, V. Muskheli, M.V. Razumova, F.S. Korte, M. Regnier, K.D. Hauch, L. Pabon, H. Reinecke, C.E. Murry, Growth of engineered human myocardium with mechanical loading and vascular coculture, Circ. Res. 109 (1) (2011) 47-59.

[16] V.F. Shimko, W.C. Claycomb, Effect of mechanical loading on three-dimensional cultures of embryonic stem cell-derived cardiomyocytes, Tissue Eng. A 14 (1) (2008) 49-58.

[17] M. Tiburcy, J.E. Hudson, P. Balfanz, S. Schlick, T. Meyer, M.L. Chang Liao, E. Levent, F. Raad, S. Zeidler, E. Wingender, J. Riegler, M. Wang, J.D. Gold, I. Kehat, E. Wettwer, U. Ravens, P. Dierickx, L.W. van Laake, M.J. Goumans, S. Khadjeh, K. Toischer, G. Hasenfuss, L.A. Couture, A. Unger, W.A. Linke, T. Araki, B. Neel, G. Keller, L. Gepstein, J.C. Wu, W.H. Zimmermann, Defined engineered human myocardium with advanced maturation for applications in heart failure modeling and repair, Circulation 135 (19) (2017) 1832-1847.

[18] W.H. Zimmermann, Tissue engineering of a differentiated cardiac muscle construct, Circ. Res. 90 (2) (2001) 223-230.

[19] G. Kensah, I. Gruh, J. Viering, H. Schumann, J. Dahlmann, H. Meyer, D. Skvorc, A. Bar, P. Akhyari, A. Heisterkamp, A. Haverich, U. Martin, A novel miniaturized multimodal bioreactor for continuous in situ assessment of bioartificial cardiac tissue during stimulation and maturation, Tissue Eng. Part C, Methods 17 (4) (2011) 463-473.

[20] O.J. Abilez, E. Tzatzalos, H. Yang, M.T. Zhao, G. Jung, A.M. Zollner, M. Tiburcy, J. Riegler, E. Matsa, P. Shukla, Y. Zhuge, T. Chour, V.C. Chen, P.W. Burridge, I. Karakikes, E. Kuhl, D. Bernstein, L.A. Couture, J.D. Gold, W.H. Zimmermann, J.C. Wu, Passive stretch induces structural and functional maturation of engineered heart muscle as predicted by computational modeling, Stem Cells 36 (2) (2018) 265-277.

[21] T. Boudou, W.R. Legant, A.B. Mu, M.A. Borochin, N. Thavandiran, M. Radisic, P.W. Zandstra, J.A. Epstein, K.B. Margulies, C.S. Chen, A microfabricated platform to measure and manipulate the mechanics of engineered cardiac microtissues, Tissue Eng Pt A 18 (9-10) (2012) 910-919.

[22] M.N. Hirt, N.A. Sorensen, L.M. Bartholdt, J. Boeddinghaus, S. Schaaf, A. Eder, I. Vollert, A. Stohr, T. Schulze, A. Witten, M. Stoll, A. Hansen, T. Eschenhagen, Increased afterload induces pathological cardiac hypertrophy: a new in vitro model, Basic Res. Cardiol. 107 (6) (2012) 307.

[23] J.L. Ruan, N.L. Tulloch, M.V. Razumova, M. Saiget, V. Muskheli, L. Pabon, H. Reinecke, M. Regnier, C.E. Murry, Mechanical stress conditioning and electrical stimulation promote contractility and force maturation of induced pluripotent stem cell-derived human cardiac tissue, Circulation 134 (20) (2016) 1557-1567.

[24] C. Ruwhof, A. van der Laarse, Mechanical stress-induced cardiac hypertrophy: mechanisms and signal transduction pathways, Cardiovasc. Res. 47 (1) (2000) 23-37.

[25] G.W. Dorn II, J. Robbins, P.H. Sugden, Phenotyping hypertrophy: eschew obfuscation, Circ. Res. 92 (11) (2003) 1171-1175.

[26] G.W. Dorn II, The fuzzy logic of physiological cardiac hypertrophy, Hypertension 49 (5) (2007) 962-970.

[27] N.J. Sniadecki, C.S. Chen, Microfabricated silicone elastomeric post arrays for measuring traction forces of adherent cells, Methods Cell Biol. 83 (2007) 313-328.

[28] N.J. Palpant, L. Pabon, C.E. Friedman, M. Roberts, B. Hadland, R.J. Zaunbrecher, I. Bernstein, Y. Zheng, C.E. Murry, Generating high-purity cardiac and endothelial derivatives from patterned mesoderm using human pluripotent stem cells, Nat. Protoc. 12 (1) (2017) 15-31.

[29] S. Schaaf, A. Eder, I. Vollert, A. Stohr, A. Hansen, T. Eschenhagen, Generation of strip-format fibrin-based engineered heart tissue, EHT, Methods Mol. Biol. 1181 (2014) 121-129.

[30] K.S. Bielawski, A. Leonard, S. Bhandari, C.E. Murry, N.J. Sniadecki, Real-time force and frequency analysis of engineered human heart tissue derived from induced pluripotent stem cells using magnetic sensing, Tissue Eng. Part C Methods 22 (10) (2016) 932-940.

[31] J.J. Chong, H. Reinecke, M. Iwata, B. Torok-Storb, A. Stempien-Otero, C.E. Murry, Progenitor cells identified by PDGFR-alpha expression in the developing and diseased human heart, Stem Cells Dev. 22 (13) (2013) 1932-1943.

[32] I.Y. Shadrin, B.W. Allen, Y. Oian, C.P. Jackman, A.L. Carlson, M.E. Juhas, N. Bursac, Cardiopatch platform enables maturation and scale-up of human pluripotent stem cell-derived engineered heart tissues, Nat. Commun. 8 (1) (2017) 1825.

[33] K. Kartasalo, R.P. Polonen, M. Ojala, J. Rasku, J. Lekkala, K. Aalto-Setala, P. Kallio, CytoSpectre: a tool for spectral analysis of oriented structures on cellular and subcellular levels, BMC Bioinformatics 16 (2015) 344.

[34] J.U. Lind, T.A. Busbee, A.D. Valentine, F.S. Pasqualini, H. Yuan, M. Yadid, S.J. Park, A. Kotikian, A.P. Nesmith, P.H. Campbell, J.J. Vlassak, J.A. Lewis, K.K. Parker, Instrumented cardiac microphysiological devices via multimaterial three-dimen sional printing, Nat. Mater. 16 (3) (2017) 303-308.

[35] R.E. Klabunde, Cardiovascular Physiology Concepts, 2nd ed., Lippincott Williams \& Wilkins/Wolters Kluwer, Philadelphia, PA, 2012.

[36] J. Ross, J.W. Covell, E.H. Sonnenblick, E. Braunwald, Contractile state of heart characterized by force-velocity relations in variably Afterloaded and Isovolumic beats, Circ. Res. 18 (2) (1966) 149.

[37] R. Beyar, S. Sideman, Model for left ventricular contraction combining the force length velocity relationship with the time varying elastance theory, Biophys. J. 45 (6) (1984) 1167-1177.

[38] A.G. Rodriguez, S.J. Han, M. Regnier, N.J. Sniadecki, Substrate stiffness increases twitch power of neonatal cardiomyocytes in correlation with changes in myofibril structure and intracellular calcium, Biophys. J. 101 (10) (2011) 2455-2464.

[39] E.H. Sonnenblick, Force-velocity relations in mammalian heart muscle, Am. J. Phys. 202 (1962) 931-939.

[40] C.F. Asnes, J.P. Marquez, E.L. Elson, T. Wakatsuki, Reconstitution of the frankStarling mechanism in engineered heart tissues, Biophys. J. 91 (5) (2006) 1800-1810.

[41] I.C. Turnbull, I. Karakikes, G.W. Serrao, P. Backeris, J.J. Lee, C. Xie, G. Senyei, R.E. Gordon, R.A. Li, F.G. Akar, R.J. Hajjar, J.S. Hulot, K.D. Costa, Advancing functional engineered cardiac tissues toward a preclinical model of human myocardium, FASEB J. 28 (2) (2014) 644-654.

[42] P. Razeghi, M.E. Young, J.L. Alcorn, C.S. Moravec, O.H. Frazier, H. Taegtmeyer, Metabolic gene expression in fetal and failing human heart, Circulation 104 (24) (2001) 2923-2931.

[43] R. Ferretti, M. Sbroggio, A. Di Savino, F. Fusella, A. Bertero, W. Michowski, G. Tarone, M. Brancaccio, Morgana and melusin: two fairies chaperoning signal transduction, Cell Cycle 10 (21) (2011) 3678-3683.

[44] H.A. Rockman, R.S. Ross, A.N. Harris, K.U. Knowlton, M.E. Steinhelper, L.J. Field, J. Ross Jr., K.R. Chien, Segregation of atrial-specific and inducible expression of an atrial natriuretic factor transgene in an in vivo murine model of cardiac hypertrophy, Proc. Natl. Acad. Sci. U. S. A. 88 (18) (1991) 8277-8281.

[45] L.B. Hazeltine, C.S. Simmons, M.R. Salick, X. Lian, M.G. Badur, W. Han, S.M. Delgado, T. Wakatsuki, W.C. Crone, B.L. Pruitt, S.P. Palecek, Effects of substrate mechanics on contractility of cardiomyocytes generated from human pluripotent stem cells, Int. J. Cell Biol. 2012 (2012) 508294.

[46] N. Hersch, B. Wolters, G. Dreissen, R. Springer, N. Kirchgessner, R. Merkel, B. Hoffmann, The constant beat: cardiomyocytes adapt their forces by equal contraction upon environmental stiffening, Biology Open 2 (3) (2013) 351-361.

[47] A.J. Ribeiro, Y.S. Ang, J.D. Fu, R.N. Rivas, T.M. Mohamed, G.C. Higgs, D. Srivastava, B.L. Pruitt, Contractility of single cardiomyocytes differentiated from pluripotent stem cells depends on physiological shape and substrate stiffness, Proc. Natl. Acad. Sci. U. S. A. 112 (41) (2015) 12705-12710.

[48] P. Bajaj, X. Tang, T.A. Saif, R. Bashir, Stiffness of the substrate influences the phenotype of embryonic chicken cardiac myocytes, J. Biomed. Mater. Res. A 95( (4) 
(2010) 1261-1269.

[49] B. Bhana, R.K. Iyer, W.L. Chen, R. Zhao, K.L. Sider, M. Likhitpanichkul,

C.A. Simmons, M. Radisic, Influence of substrate stiffness on the phenotype of heart cells, Biotechnol. Bioeng. 105 (6) (2010) 1148-1160.

[50] M.L. McCain, K.K. Parker, Mechanotransduction: the role of mechanical stress, myocyte shape, and cytoskeletal architecture on cardiac function, Pflugers Arch. 462 (1) (2011) 89-104.

[51] A.J. Engler, C. Carag-Krieger, C.P. Johnson, M. Raab, H.Y. Tang, D.W. Speicher, J.W. Sanger, J.M. Sanger, D.E. Discher, Embryonic cardiomyocytes beat best on a matrix with heart-like elasticity: scar-like rigidity inhibits beating, J. Cell Sci. 121 (Pt 22) (2008) 3794-3802.

[52] J.G. Jacot, A.D. McCulloch, J.H. Omens, Substrate stiffness affects the functional maturation of neonatal rat ventricular myocytes, Biophys. J. 95 (7) (2008) 3479-3487.

[53] S. Lee, V. Serpooshan, X. Tong, S. Venkatraman, M. Lee, J. Lee, O. Chirikian, J.C. Wu, S.M. Wu, F. Yang, Contractile force generation by 3D hiPSC-derived cardiac tissues is enhanced by rapid establishment of cellular interconnection in matrix with muscle-mimicking stiffness, Biomaterials 131 (2017) 111-120. 